\title{
Phase space structure of the hydrogen atom in a circularly polarized microwave field
}

\author{
Esther Barrabés \\ Dept. Informàtica i Matemàtica Aplicada, \\ Universitat de Girona, 17071 Girona, Spain \\ Mercè Ollé \\ Dept. de Matemàtica Aplicada I \\ Universitat Politècnica de Catalunya, Barcelona, Spain \\ Florentino Borondo \\ Departamento de Química, \\ and Instituto Mixto de Ciencias Matemáticas \\ CSIC-UAM-UC3M-UCM \\ Universidad Autónoma de Madrid, \\ Cantoblanco, 28049 Madrid, Spain \\ David Farrelly \\ Dept. of Chemistry and Biochemistry \\ Utah State University, Logan, UT 84322-0300 \\ Josep-Maria Mondelo \\ IEEC \& Dept. Matemàtiques \\ Universitat Autònoma de Barcelona, 08193 Bellaterra, Spain
}

June 10, 2011

\begin{abstract}
We consider the problem of the hydrogen atom interacting with a circularly polarized microwave field, modeled as a perturbed Kepler problem. A remarkable feature of this system is that the electron can follow what we term erratic orbits before ionizing. In an erratic orbit the electron makes multiple large distance excursions from the nucleus with each excursion being followed by a close approach to the nucleus, where the interaction is large. Here we are interested in the mechanisms that explain this observation. We find that the manifolds associated with certain hyperbolic periodic orbits may play an important role, despite the fact that, in some respects, the dynamics is almost Keplerian. A study of some relevant invariant objects is carried out for different system parameters. The con-
\end{abstract}


sequences of our findings for ionization of an electron by the external field are also discussed.

Keywords Hamiltonian dynamical systems, perturbed problem, periodic orbits, chaotic regions, ionization.

\section{Introduction}

Studies of the three body problem and, in particular, the Sun-Earth-Moon system, have stimulated an enormous number of advances in physics and mathematics, including the discovery of classical chaos [10]. Obviously chaotic dynamics, e.g., in the Solar System, can have a variety of consequences many of which might be considered negative or destructive. However, recently chaos has been implicated in the construction of various objects in the Solar System, e.g., binaries in the Kuiper belt as well as the capture of irregular moons at Jupiter and Saturn [1]. Chaos is far from being a topic restricted to classical mechanics; over the past forty years or so a vast body of literature has accumulated on the subject of chaos in microscopic, i.e., quantum, systems. Many of these studies have focussed on perturbations of the hydrogen atom which is described by the same (classical) Hamiltonian as is Kepler's problem. We mention in particular [23], [7], [4], [13], (going back 10-15 years) to the latest application [17] (and also references therein) corresponding to strong field ionization.

In classical mechanics, Hill's problem, which is essentially a perturbed Kepler problem, is widely viewed as being the paradigm of a realistic chaotic system (although other simple models certainly exist). Hill's problem can be obtained as a special case of the restricted three-body problem (RTBP). It is of note than in quantum mechanics a very close analogue to the RTBP exists, namely, the hydrogen atom interaction with a circularly polarized electromagnetic fields - which we will refer here simply as "the CP" problem (see, for example, [6] and [11]). All of the ingredients of the RTBP are contained in this system; stable or unstable motion in a rotating frame depending on a parameter; the possibility of capture or escape (of an electron); stable, unstable and chaotic motion co-existing well above all equilibrium points of the system. The latter point is significant because escape and capture are not governed by energy constraints, i.e., by surfaces of zero-velocity, but by dynamical constraints which are not immediately obvious based on a study of the potential energy surface or the surface of zero-velocity. In the $\mathrm{CP}$ problem these properties have been used to predict and simulate electronic states which are direct analogs of the Trojan asteroids in the Solar System; essentially these states are highly localized electronic wavepackets which "orbit" the nucleus in much the same way that the two groups of Trojan asteroids orbit the Sun along the same orbit as does Jupiter [5], [16].

The CP problem - as well as its extension to the case in which the field is elliptically polarized (here the EP problem) - has been the subject of intense study [11]. Nevertheless, these exhibit a variety of phenomena that remain 
poorly understood. In this article we focus, in particular, on the problem of ionization or escape of an electron in the CP problem. Because escape is related to capture by time reversal then our results can be adapted to apply to capture also.

Previous numerical simulations [6] have shown that the electron, prior to ionization, can make multiple excursions to distances which are hundreds of times the radius of the atom, then returning to the vicinity of the nucleus, before actually leaving the system (ionizing). More particularly, the behavior of the distance to the origin as a function of time can increase and decrease alternatively in an apparently random way, such that it is difficult to predict when an electron will actually ionize. Moreover, this casts doubts over the fact that ionization mechanism is adequately understood. We are interested specifically in explaining the reasons for this kind of behavior. The two main issues to be addressed in this article are (i) the structure of the stable and unstable manifolds of the problem and (ii) the mechanism by which an initially bound electron escapes through interaction of the atom with a circularly polarized microwave field.

In general, our study and objectives are similar to the earlier investigation by Simó and Stuchi [20] who studied the structure of the central stable and unstable manifolds of the Hill problem in a rotating frame, and the destruction of Kolmogorov-Arnold-Moser (KAM) tori as a function of energy. Here, we are interested in the evolution of these dynamical structures in the CP problem, and how this impacts ionization, as various physical parameters, including the energy, are varied. Our approach, however, is different and our Hamiltonian represents a microscopic system. We are particularly interested in puzzling features concerning ionizing/escaping orbits.

One approach to constructing the stable and unstable manifolds associated with saddle points - which are the gateways to escape and capture - has been to use normal form theory (NFT). However, we have discovered empirically that the NFT approach breaks down both in the RTBP and the CP problem around certain equilibrium points ( $L_{3}$ in the case of the RTBP or $L_{1}$ in the CP problem). Hence, we use a numerical alternative approach to make a systematic study of the homoclinic orbits of the CP problem.

The paper is organized in four parts which are mostly self contained but which complement each other: Section 2 is devoted to general features of the problem. The equations of motion for a hydrogen atom in rotating coordinates are given. Actually we obtain a system of ordinary differential equations (ODEs) that depends only on one parameter $K$. Also a description of the main families of periodic orbits of the problem is given. We will consider the problem from a dynamical system point of view, i.e., we will be interested in a variety of invariant objects (equilibrium points, periodic and homoclinic orbits and invariant manifolds) exhibited by this system of ODEs. In Section 3, the invariant manifolds of hyperbolic Lyapunov periodic orbits (LPO) around the equilibrium point are considered, and we also analyze the existence of homoclinic orbits to the LPO. Such homoclinic orbits allow the existence of orbits with prescribed itineraries as well as the presence of chaotic motion. In particular, transit and 
non-transit orbits are introduced. Then, in Section 4 we present a detailed description of the main features of the dynamics from a global point of view for small values of $K$. Suitable Poincaré sections are taken and the evolution of the different objects involved in the dynamics are described, when varying the energy. In particular we describe the role of invariant tori as barriers for capture and we also show some orbits suitable for ionization purposes. Therefore, we will see that there are other dynamical barriers than the zero velocity curves and the bottleneck around the unstable LPO that prevent trajectories from escaping. Some mechanisms (due to the dynamics of such invariant objects) to explain possible ionization/capture will be discussed. Finally, in Section 5 we localize and classify the set of initial conditions of ionizing and erratic orbits and we give some experimental estimates of escaping time. Conclusions are in Section 6.

We finally remark that the numerical integrations carried out along the paper have been done using a Taylor method, using the software package provided by Jorba and Zou [12]. This is a robust method, both in speed and accuracy, and suitable for long time span integrations.

\section{The CP problem}

In this Section we present the equations of the problem and some basic properties related to equilibrium points and their stability, and the zero velocity curves. Moreover, we give a description of the main families of periodic orbits (PO) that play an important role in the dynamics of the problem. In the Appendix more details with respect to changes of coordinates and others characteristics of the problem are given.

Let us consider the relative motion of a hydrogen atom subjected to a circularly polarized (CP) microwave, where the pulse of the microwave field is taken with a flat-top shape, i.e., the field amplitude is ramped up in time until it achieves a final, constant amplitude. Here we ignore the ramping and only consider the dynamics after the flat-top has been reached - see [6] for a discussion of the implications of the initial ramp.

The Hamiltonian for a hydrogen atom (in atomic units and in the limit of an infinitely massive nucleus) subjected to a CP microwave field is the following:

$$
H=\frac{1}{2}\left(p_{x}^{2}+p_{y}^{2}+p_{z}^{2}\right)-\frac{1}{r}+F(x \cos \omega t+y \sin \omega t),
$$

where $(x, y, z)$ and $\left(p_{x}, p_{y}, p_{z}\right)$ are the canonical coordinates and their conjugate momenta; $r^{2}=x^{2}+y^{2}+z^{2} ; \omega$ is the angular frequency of the microwave field and $F>0$ is the field strength (see [6]). We expect to have chaotic regions for this Hamiltonian since it is well known that a small time-periodic perturbation imposed on the Kepler problem creates a chaotic region with an erratic behavior near the parabolic orbit of the Kepler problem (see [19]).

In this paper we will consider the motion in the planar case, that is for $z=0$. Furthermore, moving to a frame rotating with the CP field and by re-scaling 
time and distances the expression of the Hamiltonian (see 6) is

$$
H=\frac{1}{2}\left(p_{x}^{2}+p_{y}^{2}\right)-x p_{y}+y p_{x}-\frac{1}{r}+K x
$$

where $K=F / w^{4 / 3}>0$ and, for simplicity, we keep the same names for the position and momentum coordinates. Now the Hamiltonian becomes autonomous and the constant value of the Hamiltonian $H=h$ over each solution is called the energy.

The CP problem in rotating coordinates has two equilibrium points, denoted by $L_{1}$ and $L_{2}$ and located in the $x$-axis at $x<0$ and $x>0$ respectively. The first one is of type saddle $\times$ center, whereas the second one is of type center $\times$ center for small values of the parameter $K$ (see the Appendix for more details about the equilibrium points). We denote by $h_{i}, i=1,2$ the energy at each equilibrium point. The richness of the dynamics around the hyperbolic point is an important key in the global dynamics of the problem. It is also important to take into account the region in configuration space where the motion is possible, the so called Hill's region. In Figure 1 the zero velocity curves (zvc) for $K=0.0015749$ and different values of the energy are shown. For other values of the parameter $K$ the curves have a similar topology. Notice that for values of the energy below that of the hyperbolic equilibrium point the region of motion has two components, whereas for bigger values of the energy, the region of motion has only one component, and the inner (around the origin) and outer regions are connected via a bottleneck region around the equilibrium point $L_{1}$. Although there exists only one component of motion for $h_{1}<h<h_{2}$, for values of the energy close to $h_{1}$ we still refer to the inner and outer regions connected via the bottleneck around $L_{1}$.
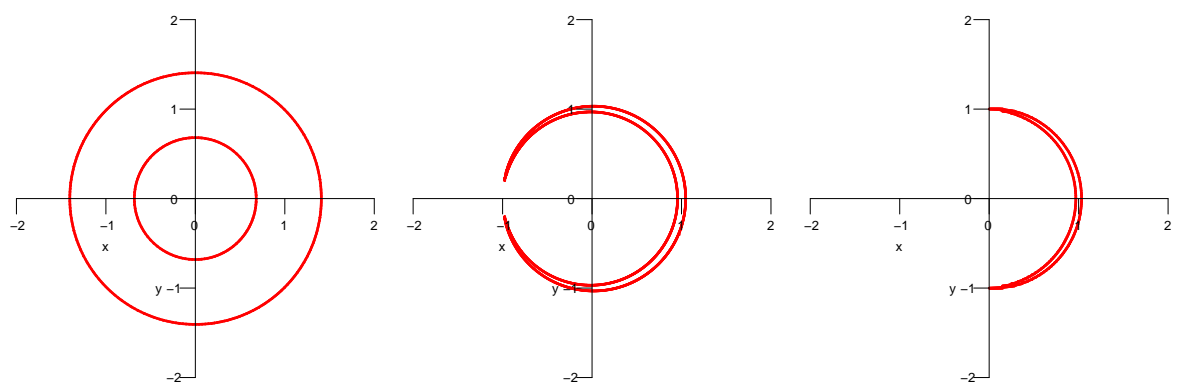

Figure 1: Zero velocity curves in $(x, y)$ coordinates of the CP problem for $K=$ 0.0015749 and the following energies: (a) $h=-1.7$ (left), (b) $h=-1.50155$ (center) and (c) $h=-1.5$ (right).

It is worth noticing that, when the particle is not far away from the nucleus, we can deal with the problem given by (2) as a perturbed Kepler problem when the parameter $K$ is small, which is the case we have considered here. In fact, we explain some features of the dynamics of the CP problem from the dynamics of 
the two body problem. We have not taken values of $K$ bigger than 0.1 (except in the computation of homoclinic connections, where a comment for the $K \geq 1$ case is made), and in general the numerics we present here are done for the values $K=0.0015749$ and $K=0.1$ (see Table 1 in the Appendix).

From the rotating two-body problem, $K=0$, we know that, for $h<-3 / 2$, there exist three families of circular POs: one family of direct orbits (denoted by $d$ ) and two families of retrograde ones (denoted by $r_{i}$ and $r_{o}$ ), see, for example [15]. Given a fixed value of $h<-3 / 2$, the $(x, y)$ projection of the PO belonging to families $d$ and $r_{i}$ lie inside the bounded Hill region, whereas the one belonging to $r_{o}$ lies in the unbounded one. When $K \neq 0$ and small, these families also exist in our problem and we denote them in the same way.

Also for $K \neq 0$ small enough, since $L_{1}$ is of type saddle $\times$ center, and $L_{2}$ of type center $\times$ center, applying the Lyapunov theorem (see [18]) when varying the energy, we obtain three families of periodic orbits (known as Lyapunov periodic orbits, LPO), one around $L_{1}\left(o l_{1}\right)$ and two around $L_{2}$; for the latter we distinguish between the families of short $\left(o l_{2 s}\right)$ and long $\left(o l_{2 l}\right)$ period (following the nomenclature of the families around $L_{4,5}$ in the RTBP). We describe briefly these six families of PO. In Figure 2 (a) we show the characteristic curves of the six families in the $\left(h, x_{0}\right)$ plane for $K=0.0015749$, where $x_{0}$ is the $x$ coordinate of the initial condition at the intersection with the $x$-axis (we have always chosen as initial condition the intersection point with biggest value of $x$ ).

- Family of direct PO $(d)$ : it exists for $h<h_{1}$, tends to the origin when $h \rightarrow-\infty$ and tends to a periodic orbit with infinity period when $h \rightarrow h_{1}$. Concerning the linear stability, the orbits are always stable with infinitely many critical orbits (that is, with the stability parameter equal to 2 or -2 ) giving rise to many bifurcated families of $\mathrm{PO}$.

- Family of inner retrograde PO $\left(r_{i}\right)$ : it exists for all values of the energy (both positive and negative). Along the family, the period of the orbits varies in $[0,2 \pi]$. There are two limiting periodic orbits: one of infinite radius and period $2 \pi$ when $h \rightarrow+\infty$, and the origin for $h \rightarrow-\infty$. The orbits are always linearly stable.

- Family of outer retrograde PO $\left(r_{o}\right)$ : it exists for $h<h_{1}$, the stability parameter varies between 2 and -2 and there appear many bifurcating families along it.

- Family of LPO around $L_{1}\left(o l_{1}\right)$ : it exists for $h \geq h_{1}$. The orbits computed are unstable. Actually, for $h$ bigger than and near $h_{1}$, they inherit the hyperbolicity of the $L_{1}$ point which is of type center $\times$ saddle. We have stopped the continuation of the family when the orbit is close to a collision with the origin. We might continue it using regularized coordinates and the PO would have a loop for energy values bigger than the one for which there is a collision orbit.

- Family of LPO of long period around $L_{2}\left(o l_{2 l}\right)$ : it exists for $h_{1}<h \leq h_{2}$ and the orbits computed are linearly stable with many critical orbits. The 
orbits follow a path in configuration space that surrounds the zvc.

- Family of LPO of short period around $L_{2}\left(o l_{2 s}\right)$ : it exists for $h \geq h_{2}$ and the orbits computed are linearly stable. We have also stopped the continuation of the family when the orbit has a collision with the origin.
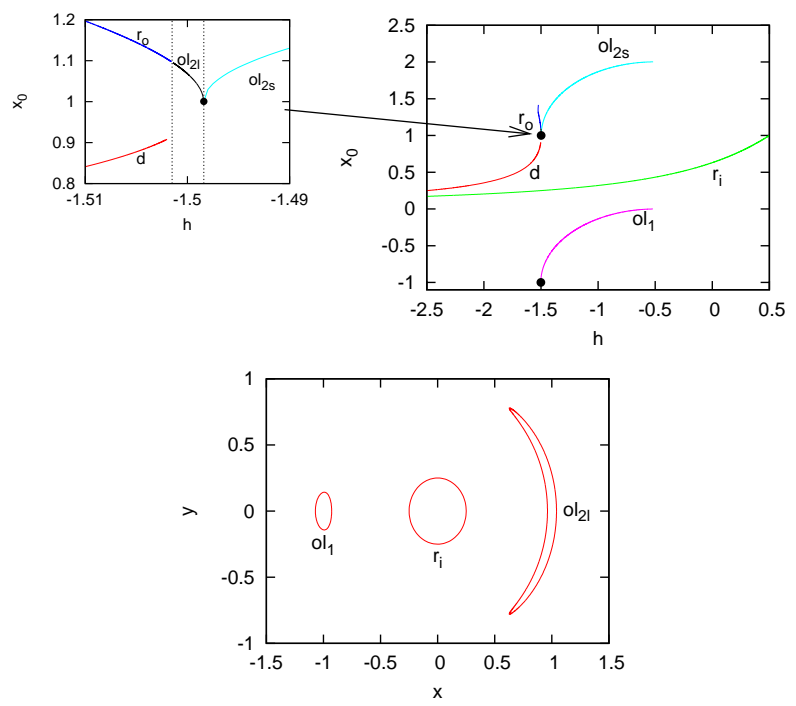

Figure 2: $K=0.0015749$. (a) Left: Characteristic curves of the six main families of periodic orbits in the $\left(h, x_{0}\right)$ plane. The black points show the location of the equilibrium points $L_{1}, L_{2}$. The vertical dotted lines show the energy level $h=h_{1}$ and $h=h_{2}$. A zoom around the equilibrium point $L_{2}$ is also included. (b) Right: For $h=-1.499$, example of a PO $(x, y)$ projection) in the family $o l_{1}$ (left orbit), one in $r_{i}$ (central orbit) and one in $o_{2 l}$ (right orbit).

Notice that the range of values of the energy between $h_{1}$ and $h_{2}$ (when the family $o l_{2 l}$ exists) can be very small, depending on the value of $K$. See Table 1.

While the zero velocity curves are closed $\left(h \leq h_{1}\right)$, the dynamics inside the bounded region of motion is strongly dominated by the two main families of periodic orbits and their bifurcations. For the study of the evolution of these bifurcations and the dynamics inside the Hill's region, we refer to [20], where a perturbed two body problem similar to the one considered here is analyzed in more detail.

\section{Lyapunov periodic orbits and homoclinic phe- nomena}

The purpose of this Section is to describe the dynamics around the hyperbolic equilibrium points and Lyapunov periodic orbits, by means of the behavior of the 
invariant manifolds, the existence of homoclinic connections and transit/nontransit orbits.

As stated above, for $h>h_{1}$, the Hill region of motion has only one component and the LPO around $L_{1}$ are unstable. So we can consider the unstable $W^{u}$ and stable $W^{s}$ manifolds associated with them. These invariant manifolds and the existence of homoclinic connections to the Lyapunov orbit are responsible for the dynamics close to the neck around the location of $L_{1}$, and of the existence of transit orbits from the inner region close the origin and the outer region, forward and backward in time.

For $h=h_{1}$, in the linear approximation of the flow around the equilibrium point $L_{1}$, a branch of the unstable 1D-manifold of $L_{1}$ departs to the $\{x<$ $\left.x_{1}, y>0\right\}$ region, and the other one departs to the $\left\{x>x_{1}, y<0\right\}$ region. We denote these branches as $W_{-}^{u}, W_{+}^{u}$, respectively, and by symmetry we have that the branches $W_{-}^{s}, W_{+}^{s}$, enter to the $\left\{x<x_{1}, y<0\right\},\left\{x>x_{1}, y>0\right\}$ regions, respectively. This was shown by [8] for the RTBP. In our problem, we have the same kind of behavior, and we have used the same notation. These branches may be regarded as the skeleton of the 2D-manifolds of the LPO for $h>h_{1}$. So we keep the same notation for the corresponding four branches of the 2D-manifolds of a LPO.

Concerning homoclinic phenomena, we recall that an homoclinic connection to an invariant object is an orbit that tends, forward and backward in time, to the invariant object. Therefore, it belongs to both its stable and unstable manifolds. We explore the existence of 'transversal' homoclinic connections that occur when the unstable and the stable manifolds of a given hyperbolic object (the equilibrium point or a LPO) intersects transversally. This implies the existence of infinitely many homoclinic connections and infinitely many periodic orbits with increasing period tending to these homoclinic connections (the so called blue sky catastrophe phenomenon by Devaney, see [9]).

Furthermore, when the invariant manifolds intersect transversally, it is possible to find orbits following a prescribed itinerary of transit and non-transit across the bottleneck around the LPO. Fixed an energy level, transit orbits, are trajectories that cross the bottleneck region determined by the zero velocity curves, going from the $\left\{x<x_{1}\right\}$ half space to the $\left\{x>x_{1}\right\}$ one or vice-versa, and non-transit orbits are the ones bouncing back to any of the half-spaces. The existence of transit and non-transit orbits is related to the behavior of the invariant manifolds and the existence of homoclinic connections. When the invariant manifolds intersect, it is possible to find orbits that alternatively live inside the inner region close to the origin and go to the outer region. More specifically, for small values of $K$ and values of the energy $h_{1}<h<h_{2}$ there exist orbits that leave the inner region, and, theoretically, should ionize (or escape, according to the transition state theory -TST-) but they do not, since they surround the exterior region, enter the interior region again, leave it, and enter again, many times as desired. Moreover, we remark that, depending on the value of the energy and $K$, the ionization is not allowed due to the existence of invariant tori that prevent from escape, as we will see in the next Section.

The existence of homoclinic connections depends on the energy level, but 
also on the value of $K$. We will focuss our attention on small values of $K$, for which we have not seen any remarkable difference, so we show here some numeric results for $K=0.1$. Some comments for big values of $K$ are included.

\subsection{Homoclinic connections to $L_{1}$}

We start considering $L_{1}$ and the existence of homoclinic connections to it. By Lerman (see [14]), we know that for any value of $K$ such that there is a symmetric homoclinic connection to $L_{1}$, there will be homoclinic connections to LPO for the values of the energy close to that of $L_{1}$. As mentioned above, this implies infinitely many connections to the same LPO, many different trajectories with prescribed itineraries and the presence of chaos.

The existence of symmetric homoclinic orbits is investigated, which occur when $W^{u}$ and $W^{s}$ intersect at the $x$-axis perpendicularly (that is, with $x^{\prime}=0$ ). In order to find the values of $K$ such that $L_{1}$ has such a connection, we follow the branch $W_{+}^{u}$ up to its $j$ intersection with the $x$ axis and we keep the value of $x^{\prime}$, that we denote by $x_{j}^{\prime}(K)$. Due to the symmetry (6), for each value of $K$ such that the function $x_{j}^{\prime}(K)=0$, the corresponding trajectory has an orthogonal crossing to the $x$-axis and becomes a symmetric homoclinic connection to $L_{1}$. Such a connection is in the "inner" region of the motion around the origin, we call it an inner connection. In order to explore "outer" connections, the intersection of the branches $W_{-}^{u}$ and $W_{-}^{s}$ should be considered. In the same way that we show that there exist an infinity of values of $K$ for which an inner homoclinic connection exists, a similar result follows for the outer connections.

For different values of $j$, we have computed the function $x_{j}^{\prime}(K)$, for $K>$ 0 . We have not found any value of $K$ such that $x_{1}^{\prime}(K)=0$, whereas there appears a decreasing sequence of values of $K$ with $x_{2}^{\prime}(K)=0$ (see Figure 3 (a)). Thus, there are many values of $K$ (tending to zero) for which a symmetric homoclinic connection to $L_{1}$ exists. These connections perform two loops around the origin, which are called 2-round homoclinics. It is worth remarking at this point the $n$-round bifurcation phenomenon that takes place at $L_{1}$ regarded as a center $\times$ saddle equilibrium point of an analytic Hamiltonian system of 2 degrees of freedom. Any $K$ belonging to a 2-round symmetric homoclinic connection is the accumulation point of $2 j$-round homoclinic connections. For a similar behavior in the RTBP see [2].

As we can see in Figure 3 (a), for small values of $K$ the values of $x_{2}^{\prime}(K)$ are also small. Therefore, the branches of the invariant manifolds are almost coincident. Even when there is no homoclinic connection to the equilibrium point, as the energy increases the manifolds of the Lyapunov orbits get bigger (in size) and for energies very close to $h_{1}$ there always exist a homoclinic connection to the corresponding orbit. This is shown in the next Subsection.

\subsection{Homoclinic connections to LPO and transit orbits}

Along this subsection we take a LPO and $W_{ \pm}^{u / s}$ are the branches of the invariant manifolds associated with it (where the sign \pm has the same meaning as in the 

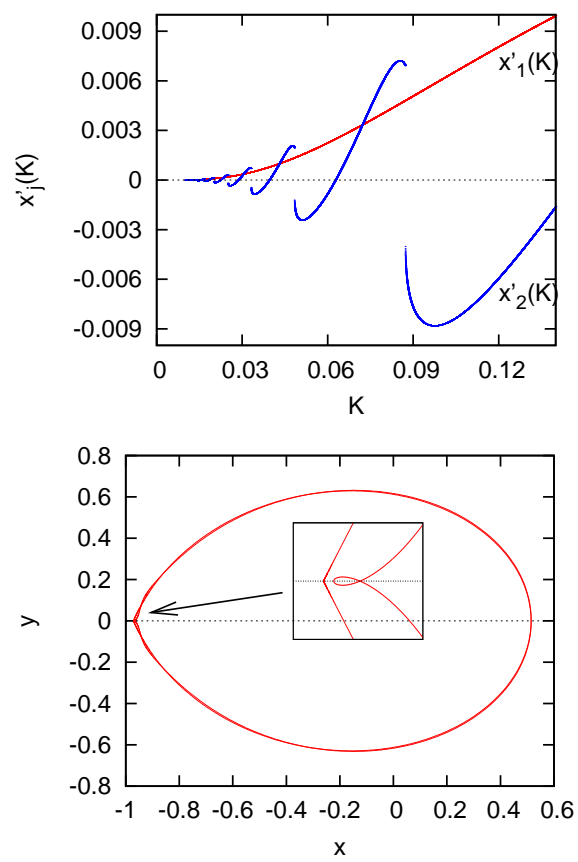

Figure 3: (a) Left. Plot of functions $x_{j}^{\prime}(K), j=1,2$, corresponding, for each $K$, to the value of the coordinate $x^{\prime}$ of the point of the orbit of the unstable invariant manifold of $L_{1}$ at its $j$-th intersection with $x$-axis. Each value of $K$ for which $x_{2}^{\prime}(K)=0$ corresponds to a 2-round symmetric homoclinic orbit (SHO). (b) Right: Example of a 2-round SHO $((x, y)$ projection) for $K=0.0880305$.

previous case). Due to the behavior of the manifolds, for values of the energy $h>h_{1}$, close to $h_{1}$, the branches $W_{+}^{u / s}$ go to the inner region limited by the zero velocity curve around the origin, whereas the branches $W_{-}^{u / s}$ go to the outer region, revolving around the zero velocity curve (see Figure 1 and 4 (ac)). Therefore, we will find homoclinic connections when the branches $W_{+}^{u / s}$ or the branches $W_{-}^{u / s}$ intersect. We denote by $u_{ \pm}^{n}=W_{ \pm}^{u} \cap \Sigma^{n}$ and $s_{ \pm}^{n}=W_{ \pm}^{s} \cap \Sigma^{n}$ the intersection of the branches of the invariant manifolds with a given Poincaré section $\Sigma$ at the $n$-th crossing. We say that there exists a homoclinic orbit of type $(-n,-k)$ if $u_{-}^{n} \cap s_{-}^{k} \neq \emptyset$, and of type $(+n,+k)$ if $u_{+}^{n} \cap s_{+}^{k} \neq \emptyset$ (see Figure 4).

We remark that with such a definition, we may have either symmetric or non symmetric homoclinic orbits. Notice that homoclinics of type $(+n,+m)$ perform a path in the inner region limited by the zero velocity curve, whereas that of type $(-n,-m)$ revolve around the zero velocity curves in the outer region.

We only present some of the homoclinics found for small values of $K$. A whole description of the families of homoclinic connections when the parameter 
$K$ varies is beyond the scope of this paper. In the figures that follow in this section, red color corresponds to unstable manifolds and blue to stable ones. The section considered has been $\Sigma=\{x=a\}$, where $a$ is a fixed and suitable value depending on the energy, the branches taken into account and the value of $K$. The branches of the invariant manifolds are 2-dimensional sets, and thus their intersections with a specific section, the sets $u_{ \pm}^{n}$ and $s_{ \pm}^{n}$, are 1-dimensional sets. As the energy is constant and the value of the $x$ coordinate is fixed, we have plotted these intersections in the plane $\left(y, p_{y}\right)$ (see Figure $4,(\mathrm{~d}-\mathrm{f})$ ).
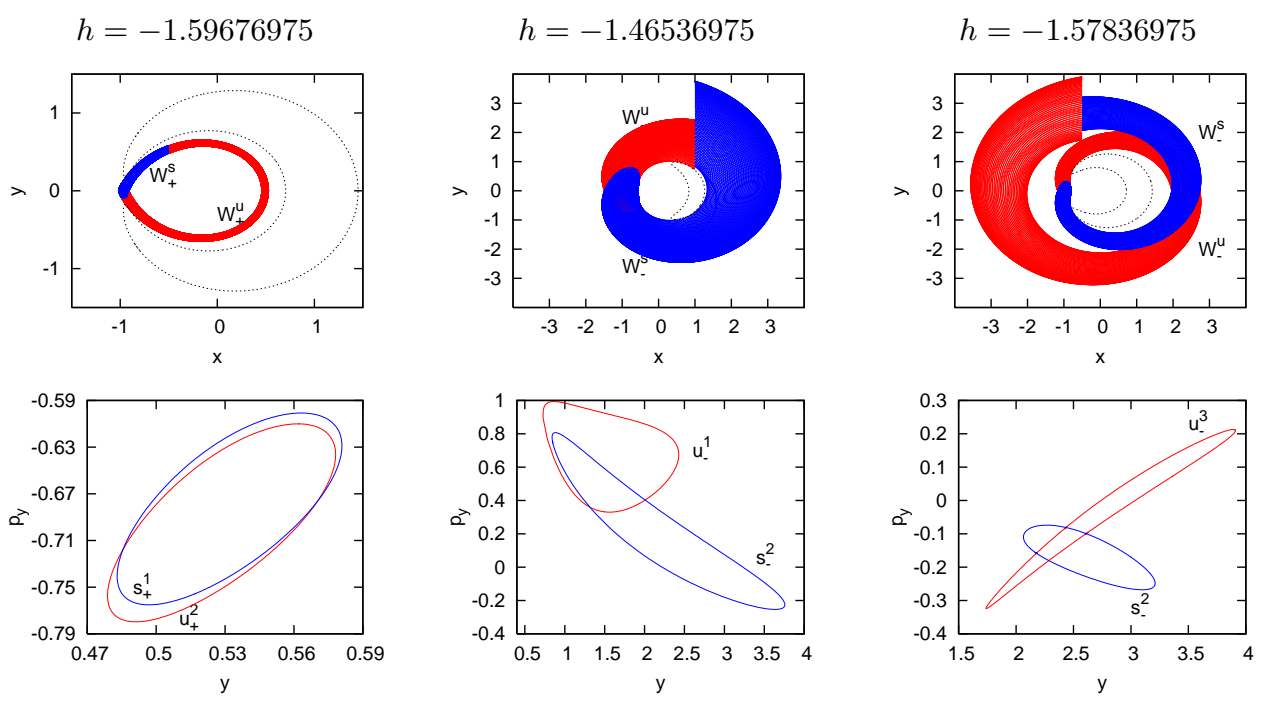

Figure 4: $K=0.1$. The values of the energy are indicated above each column. Top: from left to right (a), (b), (c), the branches of the invariant manifolds associated to a Lyapunov orbit around $L_{1}$ in configuration space. The dotted lines represent the zvc at each energy level. Bottom: from left to right (d), (e), (f), the sets $u_{ \pm}^{n}, s_{ \pm}^{k}$ corresponding to the intersection of the invariant manifolds of the first row with a specific section, plotted in the plane $\left(y, p_{y}\right)$. The intersection points of these sets correspond to homoclinic connections of type $(+2,+1)$ (left, (d)), (-1, -2) (middle, (e)), and $(-3,-2)$ (right, (f)).

We show the results obtained for $K=0.1$. The inner homoclinics are obtained when the branches $W_{+}^{u}$ and $W_{+}^{s}$ are considered (see Figure 4, left (a) and (d)). Starting with their second and first intersections respectively with a section $\Sigma=\{x=a\}$, for a constant $a \in\left[x_{1}, 0\right]$, we have found that both branches intersect, for any value of the energy $h>h_{1}$ (as we expected before the exploration done in the previous Subsection). Thus there exist at least two families of inner homoclinic connections of type $(+2,+1)$ for a certain range of values of the energy $h>h_{1}$. See Figure 5, (a), for an example of such a homoclinic orbit.

Concerning outer homoclinics, we study the behavior of the branches $W_{-}^{u}$ 
and $W_{-}^{s}$ up to several intersections with a section $\Sigma$. Close to the equilibrium point (i.e., considering LPO with energy close to that $h_{1}$ ), these branches do not intersect. As the energy increases, the invariant manifolds get wider and they intersect giving rise to the first outer homoclinics (see Figure 4, center (b), (e) and right (c), (f)). We have found homoclinics of type $(-1,-2)$ for $h \geq-1.54736975$ and of type $(-3,-2)$ for $h \geq-1.59236975$. In Figure 5, (b) and (c), two examples of these homoclinic connections are shown.

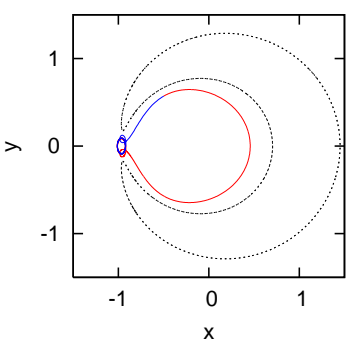

$(+2,+1)$

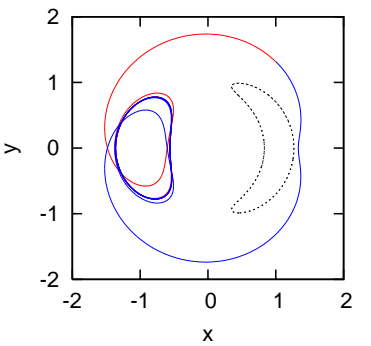

$(-1,-2)$

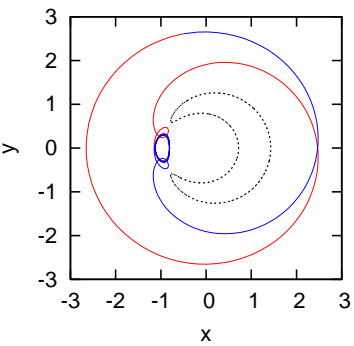

$(-3,-2)$

Figure 5: $K=0.1$. Examples of homoclinic connections in configuration space, (a) an inner homoclinic (left, $h=-1.59676975)$, and two outer homoclinics ((b) middle and (c) right, $h=-1.46536975$ and $h=-1.57836975)$, each one of the type indicated below. The dotted lines represent the zvc at each energy level.

Concerning the existence of homoclinic orbits for different values of the parameter $K$, we remark the following:

1. We have found homoclinic orbits of type $(+2,+1)$ (inner homoclinics, see Figure $4(\mathrm{~d}))$ for all values of the energy $h>h_{1}$ explored and for the values $K=0.1,1,2,5,10$. We expect to have such kind of homoclinics for all values of $K$ (at least within the range $[0,10]$ ).

2. We have not found homoclinic orbits of type $(-n,-k)$ (outer homoclinics) for $K=2,5,10$ and values $n \leq 15$ and $k \leq 14$, whereas for the values of $K \leq 1$ considered there exist homoclinic orbits of type $(-n,-k)$ for different values of $n$ and $k$ (see Figure 4 (e) and (f)).

3. In this paper, just particular homoclinic orbits of several types have been computed, but we might also be interested in computing whole families of homoclinic orbits to periodic orbits. The numerical method for such a computation and the application to the RTBP (in celestial mechanics) are described in [3].

As stated above, the existence of a homoclinic orbit implies the existence of transit orbits. When the unstable invariant manifold $W_{-}^{u}$ intersects the stable one, part of it goes "inside" the tube $W_{-}^{s}$ (see the part of the red curves inside the blue ones in Figure 4 (d-f)), returns close to the LPO orbit in the bottleneck region and travels to the inner region (with respect the $\mathrm{zvc}$ ) of motion: at this 
passage close to the LPO the orbits are transit orbits (see Figure 6 (a)). If the orbit considered does not go "inside" the tube of the stable manifold, the next approach close the LPO will be a non-transit passage (see Figure 6 (b)). Analogously, as $W_{+}^{u}$ intersects $W_{+}^{s}$, if an orbit goes "inside" the tube $W_{+}^{s}$, it will be a transit orbit from the inner region towards the outer region. An so forth: an orbit on one branch of the invariant manifolds can travel from the outer to the inner regions or viceversa, depending on its position with respect the successive intersections between the invariant manifolds. Here we use the term transit for an orbit that travels from the inner region around the nucleus to the outer region (or viceversa) at least once, and non-transit otherwise.
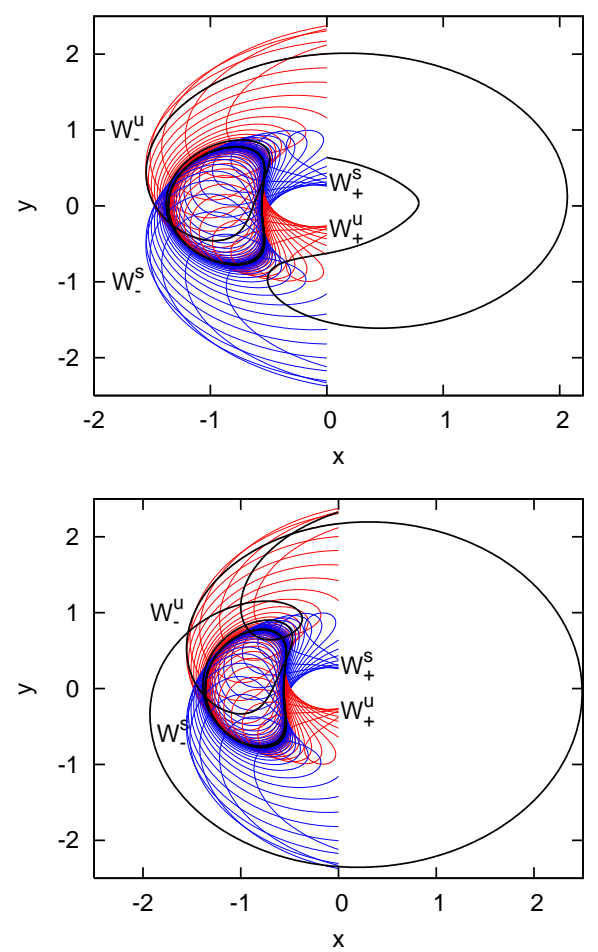

Figure 6: Example of transit ((a), left) and non-transit ((b), right) passages. The orbit on the left plot (a) has a transit passage towards a neighborhood of the origin at its approach to the Lyapunov orbit, whereas the orbit on the right plot (b) does not. In both cases, the orbits (in black) belong to the unstable branch $W_{-}^{u}$ which intersects the stable branch $W_{-}^{s}$.

This fact has special interest when the invariant manifolds are not confined inside a bounded region (we explain this in more detail in the next Section). In Figure 7 an example of an orbit with transit passages belonging to $W_{-}^{u}$ is shown. We plot the $(x, y)$ projection and the evolution of the distance to the origin, $r(t)$, when varying the time. In this specific example, the orbit has 
two passages through the bottleneck around the LPO, but it might have other close approaches to the LPO for bigger values of time. The irregular oscillating pattern of $r(t)$ is well explained from the transist/nontransit behavior of the orbit.
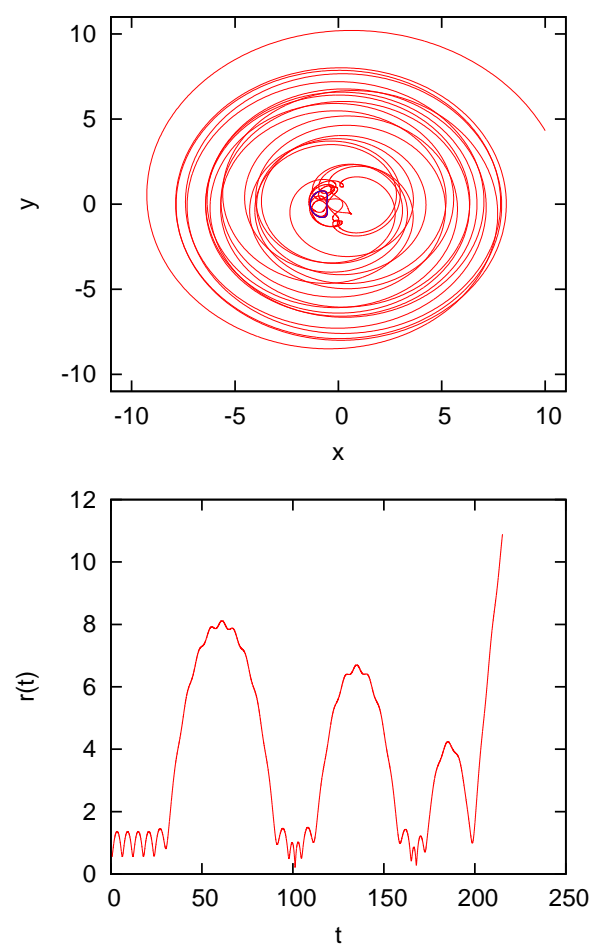

Figure $7: K=0.1, h=-1.46536975$. Example of an ionizing orbit belonging to the unstable branch $W_{-}^{u}$ with transit passages close to the LPO. (a) Left, projection in configuration space of the orbit and the LPO (in blue). (b) Right, curve of the distance to the origin as a function of time, $(t, r(t))$.

As we have seen, the transit and non-transit orbits are due to the existence of basic homoclinic connections, which correspond to the first intersections between the branches of the invariant manifolds. Furthermore, this existence also implies the existence of infinitely many other homoclinic connections. In order to illustrate this infinity, we have considered the LPO, for $K=0.1$ and $h=-1.46536975$ in Figure $5(\mathrm{~b})$, parameterized with an angle $\theta \in[0,2 \pi]$. For each $\theta$ we have considered the associated orbit on $W_{-}^{u}$, and we have computed the necessary time to reach the Poincaré section $x=1$ after $\mathrm{n}$ iterates, $t_{n}^{x=1}$. Each homoclinic connection corresponds to a value $\theta$ for which there exists a value of $\mathrm{n}$ such that $t_{n}^{x=1}$ is not defined. On the one hand, we show in Figure 8 (a) the curves $\left(\theta, t_{n}^{x=1}\right)$, for $n=3,5,7,9$. For $n=3$ we can see the two particular values of $\theta$ corresponding to the two homoclinic orbits of type $(-1,-2)$ (see 
also Figure $4(\mathrm{e}))$. For $t_{n}^{x=1}$, with $n=5,7$ we also see many other values of $\theta$ associated with homoclinic orbits of other types. Of course, this rich structure repeats on and on for higher values of $n$. On the other hand, in order to detect ionizing orbits belonging to the invariant manifolds, we plot in Figure 8 (b), the curves $\left(\theta, t_{1}^{x=5}\right)$ and $\left(\theta, t_{1}^{x=10}\right)$, that is, the time to reach the section $x=5$ and the one to reach $x=10$, assuming this time is less than a bounded time $t_{\max }$ $\left(t_{\max }=130\right.$ in this computation). If an orbit does not reach these values of $x$ it is considered as a confined orbit. The values of $\theta$ in the plot that have no image on the curves correspond either to orbits that are confined for all time in a region $(x, y)$ with $x<5$ (or $x<10$ ), or to orbits that need a time longer than $t_{\max }$ to reach the given section. On the contrary, the orbits that reach "faster" the sections fixed are the candidates to be orbits that ionize. As we can see, there are many different kind of orbits, and in particular many candidates of orbits on the unstable manifold to escape to infinity, with transits and no-transits.

\section{Ionizing/escaping orbits}

One of the main goals of the paper is to localize and explain the behavior of orbits that ionize "slowly", in the sense that they do escape towards infinity after a complicated dynamical history. We will see that we can find two types of these orbits: ones that are essentially perturbed keplerian ellipses, and other due to the behavior of the invariant manifolds associated to the LPO, like the ones we have shown before depending whether they exhibit transit passages to the origin or not.

In this Section we describe, by means of Poincaré section plots (PSP), the evolution, when varying the energy $h$, of the dynamics from a global point of view, taking into account the different invariant objects that take place and their role. In particular, we begin recalling the dynamics of the two-body problem and then we analyze its evolution for $K>0$, in particular the destruction of the invariant KAM tori that give rise to chains of periodic islands and chaotic zones.

A PSP has been typically computed as follows: for a fixed value of the energy $h$ we take a set of initial conditions $x=x_{0}, y=0, x^{\prime}=0$ and $y^{\prime}=y_{0}$ $\left(\left|y_{0}\right|\right.$ obtained from the energy $\left.h\right)$ and varying $x_{0}$. From the two-body problem $(K=0)$, one can see if the initial conditions considered correspond to an approximated hyperbolic orbit (positive energy in the non-rotating frame) or to an approximated elliptic orbit (negative energy). It can be shown (see next Section) that for a positive $x$ bigger than a certain value, all the values $y_{0}^{\prime}>0$ correspond to approximated hyperbolic orbits, which simply spiral outwards and escape towards infinity. Therefore, we have always considered $y_{0}^{\prime}<0$. For each initial condition, we integrate the corresponding orbit up to $n$ intersections with the section $x^{\prime}=0$-we will simply say $n$ iterates- and we plot the $(x, y)$ projection of the $n$ intersection points such that $y^{\prime}<0$. 

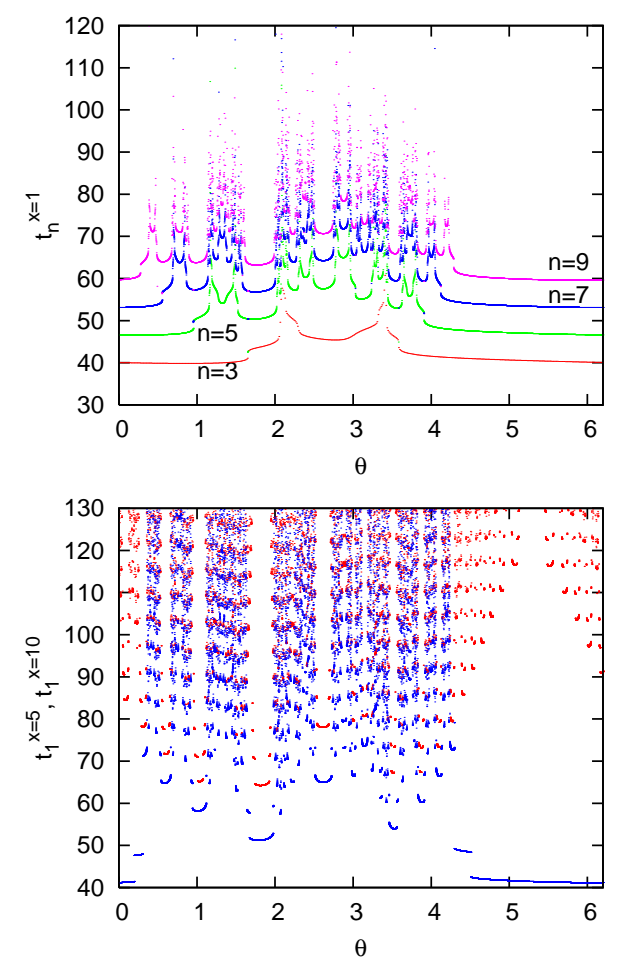

Figure 8: $K=0.1, h=-1.46536975$. We plot $\left(\theta, t_{n}^{x=a}\right)$, where $\theta$ is the angle used to parameterized the LPO and $t_{n}^{x=a}$ is the time needed by the associated orbit to $\theta$ on the unstable branch $W_{-}^{u}$ to reach the Poincaré section $x=a$ after $n$ iterates. (a) Left, section $x=1$ and $n=3,5,7,9$. To each $\theta$ such that $t_{n}^{x=a}$ is not defined corresponds a homoclinic orbit. (b) Right. $\left(\theta, t_{1}^{x=5}\right)$ (blue) and $\left(\theta, t_{1}^{x=10}\right)$ (red). In this case, the values of $\theta$ without image correspond either to bounded orbits or orbits that need a larger time than $t=130$ to reach the section $x=5$ or $x=10$.

\subsection{The two-body problem: $K=0$}

It will be useful to recall first the case $K=0$, that is the rotating two-body problem. As it is well known, this is an integrable problem and the possible orbits come simply from the rotation of ellipses, parabolas or hyperbolas. Therefore, the bounded orbits correspond to rotating ellipses which are either periodic or quasiperiodic orbits, seen as fixed points or invariant curves in the PSP. In Figure 9, we show a PSP for $K=0$ and a fixed value of the energy, with some of the invariant curves corresponding to rotating ellipses and the escaping points corresponding to a parabolic and a hyperbolic orbit. On one hand, there are invariant curves around the origin corresponding to orbits inside the bounded component of Hill's region (see Figure 1, (a)), on the other hand, there are in- 
variant curves corresponding to rotating ellipses in the unbounded component of the Hill's region. Such invariant curves are responsible for the inverted bell shape of the PSP (in blue in Figure 9). It is clear that the escaping orbits correspond to parabolic or hyperbolic orbits. The limit (or boundary) between the bounded and the unbounded orbits corresponds to the parabolic orbit that exists for each fixed value of the energy.

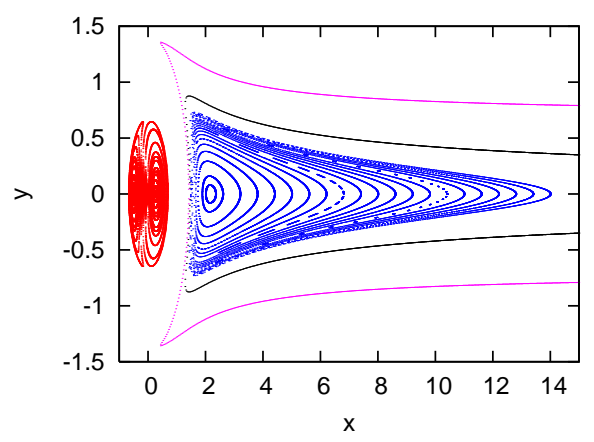

Figure 9: $K=0$ and $h=-1.7$. Points $(x, y)$ on the PSP $x^{\prime}=0, y^{\prime}<0$. For the rotating Kepler problem, the orbits can only be either rotating ellipses (the invariant curves in the plot, the red ones in the bounded component of the Hill region and the blue ones in the unbounded Hill region) or rotating parabolas or rotating hyperbolas (both cases correspond to unbounded sets on the PSP). The parabolic orbits are the frontier between the elliptic and hyperbolic ones.

\subsection{Global dynamics for $K>0$}

Now we consider our problem for $K \neq 0$, and we start considering the value $K=0.0015749$. We choose this value as the one corresponding to $F=0.01$ and $w=4$ used in Figure 12 in [6]. In Table 1 the location of the equilibrium points and its energy are shown. Since the Hamiltonian is non-integrable, the dynamics is richer and there appear other invariant objects typically expected in Hamiltonian systems with 2 degrees of freedom. Actually the generic character of motion in such systems is now fairly well understood. In particular, many invariant curves survive and many others are destroyed (according to KAM theorem) and there appears the overlapping of resonances phenomenon. So the regular orbits and chaotic ones are intimately interlaced.

Of course we will find such dynamics in our Hamiltonian. What we want to stress is the inheritance of the $K=0$ case as well as to identify some of the invariant objects that appear for different values of the energy. In particular, for $K>0$ the PSP keeps its inverted bell shape. Moreover, we focus our attention on the main purpose in this paper, which is the explanation and location of erratic orbits: orbits that seem to ionize, but do not, or that ionize very slowly (large escaping time). Typically, the $(x, y)$ projection of these orbits can travel 
close to (depending on the zvc barrier) and very far from the origin in an erratic way, that is, the distance $r(t)=\sqrt{x(t)^{2}+y(t)^{2}}$ has several different maxima and minima, with large differences between these extrema. Of course the minimum distance will depend on either the zvc barrier or the last invariant curve (when varying the energy).

With these aims in mind, next, we analyze for different values of the energy the PSP obtained. We explore the following ranges of $h$ :

1. $h<h_{1}$. In this case the forbidden Hill's region is a ring whose boundaries are two closed curves (see Figure 1 (a)). Therefore the motion in configuration space takes place either in the inner region (or bounded component) or in the outer region (unbounded component).

So let us take $h=-1.7$ (from a qualitative point of view we have the same dynamics for smaller values of $h$ ). In Figure 10 a PSP for this energy level is shown. The problem behaves as the rotating 2-body problem in the bounded (and small) possible Hill's region of motion around the origin (see Figure 10, (a)). We clearly see two stable fixed points that correspond to the stable direct orbit of family $d$ with $x_{0}>0$ and the stable retrograde one of family $r_{i}$ with $x_{0}<0$, respectively. We also appreciate the invariant curves surrounding them. Notice the similarity with the left hand side of Figure 9 .

On the unbounded Hill's component, we can see the stable retrograde periodic orbit of family $r_{o}$ and the invariant curves surrounding it (Figure 10, (b)). We remark the differences with the $K=0$ case on the unbounded Hill component, as the dynamics is not so regular due to the destruction of some of the invariant curves from $K=0$ to $K>0$. There are many periodic orbits, thin chaotic layers separated by invariant curves and also regions of global stochasticity, since many external invariant curves are broken and the merging of layers occurs. Clearly, inside the invariant curves the motion is bounded. The farther from the origin the initial conditions are taken, the more the invariant curves are broken, appearing stable periodic orbits (chains of islands) and hyperbolic periodic orbits instead.

Outside these invariant curves, it is possible to find erratic orbits, visiting different chains of islands. The presence of hyperbolic orbits in between these chains of islands and heteroclinic connections between them allows the existence of orbits that escape slowly going far and close to the zvc several times. Actually, this is the typical behavior of orbits that take place outside the outermost invariant curve around the origin and are related to the chain of islands present in the chaotic zone. In spite of the apparent stochastic behavior of the iterates, we can detect a certain structure: on one hand the iterates surround several chain of islands, passing through them each time the orbit makes a turn around the origin. On the other hand, at a certain moment, the iterates start to go farther and farther from the origin at each turn around it. This kind of orbits can ionize, but 


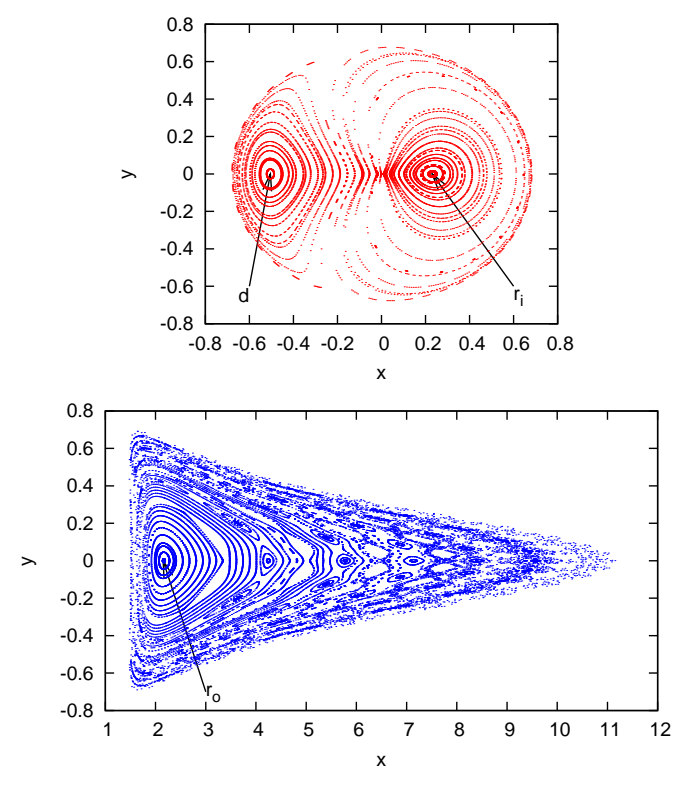

Figure 10: $K=0.0015749$ and $h=-1.7$. Points $(x, y)$ on the PSP for $x^{\prime}=$ $0, y^{\prime}<0$ (a) in the bounded component of Hill region (left), and (b) in the unbounded Hill component (right). In the inner region, the two fixed points correspond to two stable periodic orbits of the main families $d$ and $r_{i}$. In the outer region, a stable periodic orbit of the family $r_{o}$ is shown.

the time to escape definitely to infinity is greater than the escaping time for those orbits close to rotating hyperbolic orbits for which the distance to the origin increase from the very beginning.

2. $h \in\left(h_{1}, h_{2}\right)$. We fix the value $h=-1.5$ which is in the range desired for the value of $K$ considered (see Table 1). For this range of energies, the zvc is a bounded right-moon shaped curve that intersects the $x$-axis at two points (only at the point $L_{1}$, for the exceptional case $h=h_{1}$ ). See Figure 1, (b). In Figure 11 a PSP for $h=-1.5$ is shown. In this Figure the forbidden region of motion is also recognizable among the iterates of the Poincaré map in the inner region and those in the outer region. Recall that in this case there is a neck that allows trajectories traveling from the inner region (around the origin) and the outer region. Therefore there is no motion barrier (on the $(x, y)$ position variables) around the origin.

For $h=h_{1}$ the equilibrium point $L_{1}$ appears, and the inner direct orbit and the outer retrograde ones, existing for $h<h_{1}$, disappear for $h=$ $h_{1}$ tending to the 1-dimensional manifolds $W^{u}$ and $W^{s}$ of the unstable equilibrium point $L_{1}$, as stated previously. Now, for each $h>h_{1}$, on one hand there exists the LPO around $L_{1}$, as well as its invariant stable and unstable manifolds, which are almost coincident. On the other hand, 


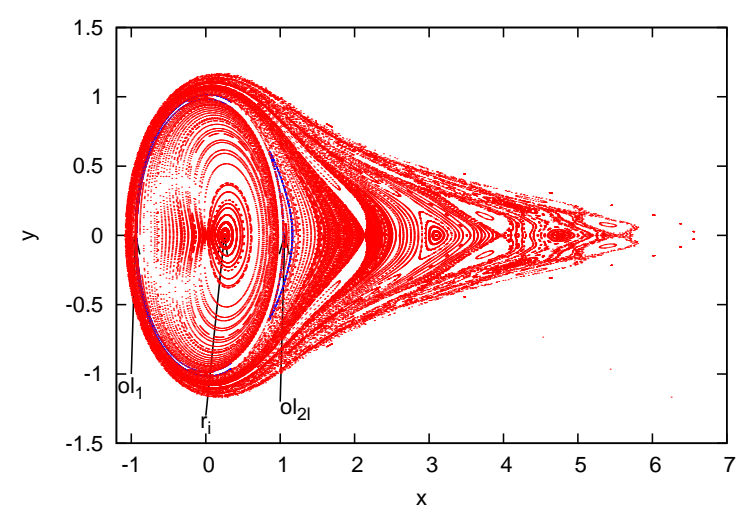

Figure 11: $K=0.0015749, h=-1.5$. PSP plot where the orbits of the main families are shown. The $1 \mathrm{D}$ curves corresponding to the intersection of the $2 \mathrm{D}$ invariant manifolds of the LPO around $L_{1}$ with the section are also shown in blue. In this case, these invariant manifolds are confined inside the outermost invariant curve around the origin. We also see the moon-shaped forbidden region of motion.

there also exists the internal retrograde periodic orbit (and the invariant curves around it) that persists for all values of the energy. And, although $L_{2}$ does not exist, the LPO of long period of family $o l_{2 l}$ does. All of these orbits of the main families $o l_{1}, r_{i}$ and $o l_{2 l}$ are shown in Figure 11.

Around the origin there is still a region of bounded orbits due to the persistence of some invariant curves that confine the motion inside them. Outside the outermost invariant curve around the origin (there are infinitely many other invariant curves farther on, grouped in chain of islands, but they do not confine the motion we are interested in) the chaoticity increases and we can find erratic orbits visiting the chains of islands and suitable for ionization.

Notice that for the energy level shown in Figure 11, the invariant manifolds $W_{-}^{s}$ and $W_{-}^{u}$ of the LPO around $L_{1}$ (in blue in the Figure) are confined inside the region bounded by the outermost invariant curve and, therefore we can conclude that for this range of values of $h$ these invariant manifolds are not responsible for ionization and do not play any role in the existence of orbits with slow ionization. Clearly, this depends on the energy value considered, for higher values of $h$ it might happen that the invariant manifolds are not confined. This will be shown for bigger values of $K$.

Since orbits suitable for ionization take place outside the last invariant curve, it could be of interest, at each energy level, to locate this last or outermost invariant curve. In order to do this, we have taken an initial condition $x_{0}$ such that it is outside the outermost (from the PSP), for 
example $x_{0}=5.5$ and $10^{5}$ iterates. Then, we plot the corresponding PSP just for this orbit. See Figure 12, where the last invariant curve is the frontier of the chaotic region.

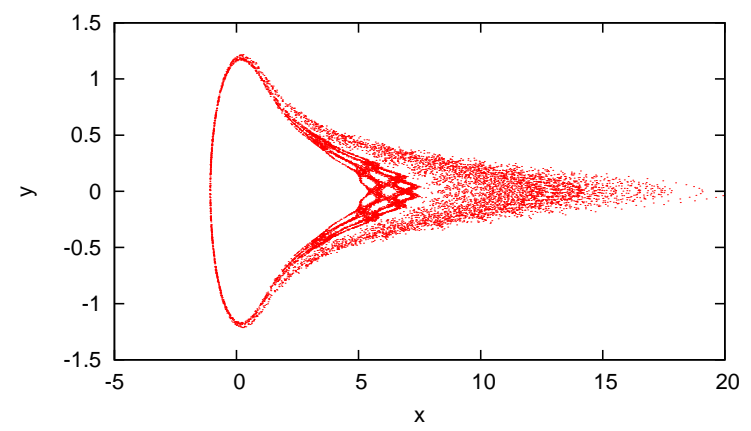

Figure 12: $K=0.0015749, h=-1.5$. Points $(x, y)$ on the PSP for the orbit $x_{0}=5.5, y=0, x^{\prime}=0, y^{\prime}<0$. The frontier of the stochastic region gives rise to the last invariant curve.

3. $h_{2}<h$. We have taken $h=-0.557$ to show the behavior of the dynamics. Now there is no zvc. There exist the stable retrograde orbit of family $r_{i}$, the stable LPO of short period around $L_{2}$, and the hyperbolic LPO around $L_{1}$ and its invariant manifolds, $W^{u}$ and $W^{s}$. In Figure 13 we show the PSP for the energy value chosen. As happened before in the previous case, at this energy level, there exists a last invariant curve around the origin. As in the previous case, for this value of $K$, the outermost confines the invariant manifolds of the LPO of family $o l_{1}$. Erratic/ionizing orbits can be found outside it.

As a final comment, we remark that some erratic orbits located on the inverted bell-shaped part of the PSP can be related to those of the $K=0$ case. In fact, this kind of erratic orbit consists of a "chain" of approximated ellipses such that at each turn around the origin there is a "close" approach to the nucleus (the minimum distance depending on the $\mathrm{zvc}$ ) and the maximum distance tends to increase (maybe not at each turn, but in general the tendency is to increase). In Figure 14 an orbit of this type is shown: (a) the PSP of the orbit, where we can see the structure shown in the previous plots; (b) the orbit in non-rotating coordinates the chain of approximated ellipses can be clearly seen; and (c) the behavior of the variations of the osculating semi-major axis and eccentricity along the orbit, that is, computed as if it were a solution of the two-body problem (see also next Section).

Now we consider bigger values of $K$. The PSP are qualitatively similar to the ones shown in the previous cases, and thus, erratic orbits can be found visiting the chaotic layers. However, the main difference is that for bigger values of $K$ and depending on the energy $h$, the invariant manifolds $W^{u}$ and $W^{s}$ of the LPO 


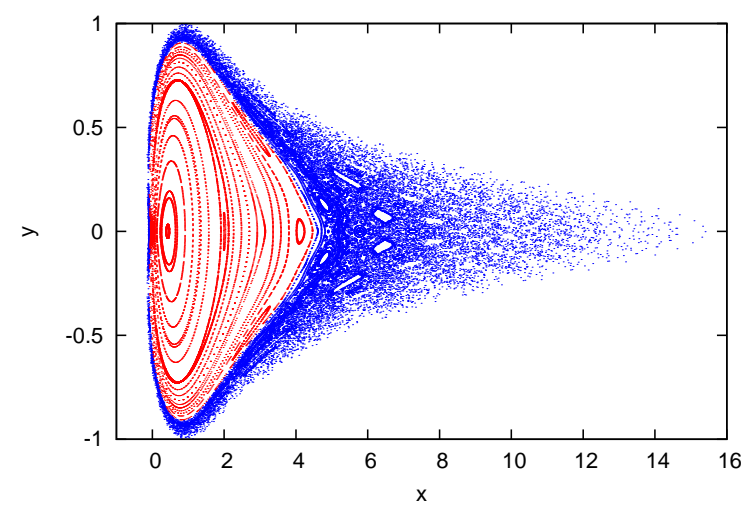

Figure 13: $K=0.0015749, h=-0.557$. Points $(x, y)$ on the PSP for $x^{\prime}=$ $0, y^{\prime}<0$. Blue and red colors correspond to orbits outside and inside the outermost invariant curve around the origin, although this invariant set has not been computed.

of the family $o l_{1}$ are not confined inside the outermost invariant curve around the origin. Therefore, they do play a role in the existence of escaping/ionizing orbits.

For example, we consider $K=0.1$ and $h=-1.46536975$, which is the value taken in the previous subsection when exploring homoclinic orbits, see Figure 4 (b) and (e). In Figure 15 we plot a PSP where we can distinguish between the region of invariant curves inside the outermost (confined motion), some erratic and hyperbolic (fast escaping) orbits, and the invariant manifold $W_{-}^{u}$ ( $W_{-}^{s}$ behaves similarly). Notice that the invariant manifolds are outside the outermost invariant curve. We have just plotted the first 30 iterates of the manifold with the Poincaré section $x^{\prime}=0, y^{\prime}<0$ but we might find orbits on $W^{u}$ that grow and escape. In this case, due to the intersection of the manifolds $W^{u}$ and $W^{s}$, their interaction is very rich and there exist infinitely many homoclinic orbits (see also the previous Section), transit and non-transit orbits confined for all time and also transit and non-transit orbits that finally escape (ionize).

Let us illustrate this rich variety of orbits. We consider now the Poincaré section $\Sigma=\{x=1\}$. In Figure 4, (b) and (e), we have shown the intersections of the outer branches of the invariant manifolds of the LPO of this energy value with the section $x=1$ at their first encounter, that is, at the first intersection of $W_{-}^{u}$ with $\Sigma$ and the second one of $W_{-}^{s}$ with $\Sigma$ giving rise to the curves $u_{-}^{1}$ and $s_{-}^{2}$, respectively. Clearly, there exist two (outer) homoclinic connections. We focuss our attention on the part of the curve $u_{-}^{1}$ (red curve in the plot) inside the region bounded by the curve $s_{-}^{2}$ (blue one). When following the flow up to the third crossing with $\Sigma$, we obtain an open curve that spirals, on and on, tending to $u_{-}^{1}$ and inside it. More concretely, the orbits on this part of the unstable branch will have (along the time from the first to the third crossing with $\Sigma$ ) a transit passage at their return close to the LPO, and after some time 

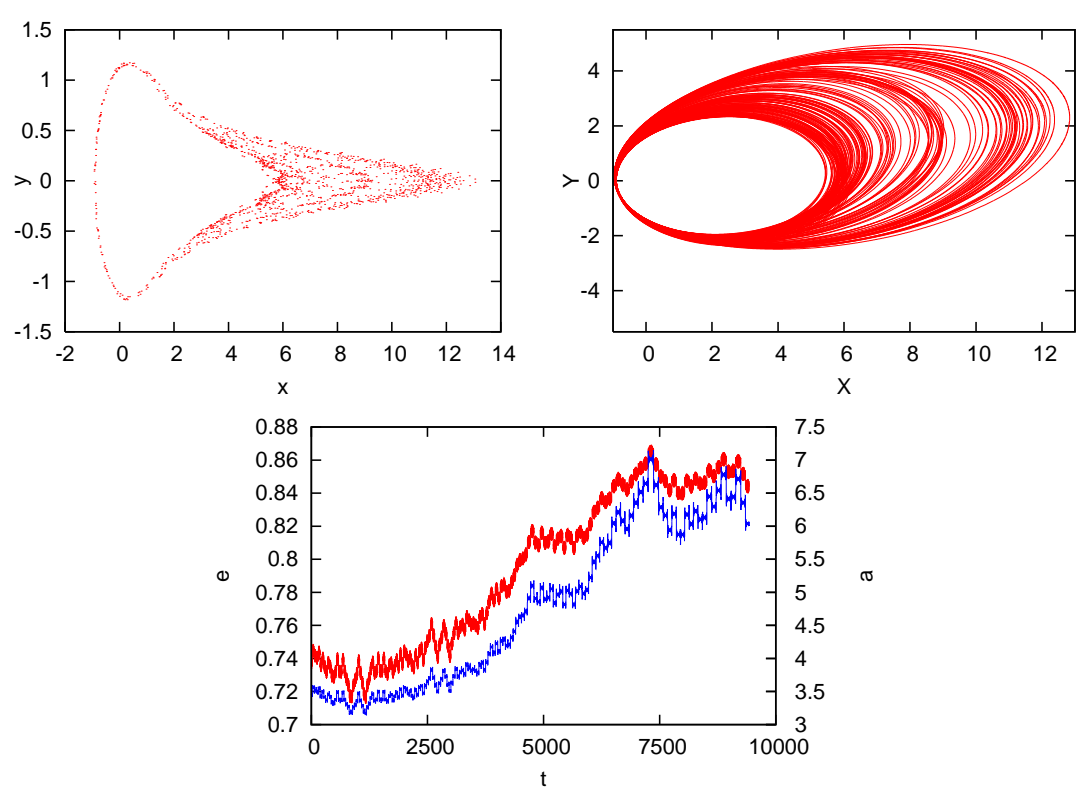

Figure 14: $K=0.0015749, h=-1.4$. We take an orbit with initial condition $x_{0}=6$., $y_{0}=x^{\prime}=0$ and $y_{0}^{\prime}<0$ and follow it for a long time. (a) Top left, PSP of the orbit. (b) Top right, the projection in configuration space of the orbit in non-rotating coordinates. In spite of the apparent chaotic behavior of the iterates on the PSP, the orbit follows different perturbed ellipses. (c) Bottom, the evolution of the semi-major axes $a$ and the eccentricity $e$ (curves $(t, e)$ in red and $(t, a)$ in blue).

around the origin, they eventually can have another transit passage to the outer region and will return to the section $x=1$ again. On the other hand, if we consider the part of the curve $u_{-}^{1}$ (red curve) outside the curve $s_{-}^{2}$ (blue one) in Figure 4, these orbits have a non-transit passage close to the LPO and return to the $x=1$ section again, giving rise to the spiraling curve to $u_{-}^{1}$ and outside it. See Figure 16 (a). Of course, when following the flow on $W_{-}^{u}$ forward in time, for example from the 3 rd to the 5 th crossing with $\Sigma$, we have the same kind of behavior, which is represented in Figure 16 (b).

Notice that, on the one hand the intersection of the unstable branch with the section (when taking into account different crossings) is broken in several curves that intersect the stable curve, giving rise to infinitely many homoclinic connections. These homoclinic connections correspond to the discontinuity points on Figure 8. Remark that in this figure, for $n=3$, also transit and non-transit orbits can be detected. Transit orbits of the unstable manifold spend more time in their return to the section $x=1$ and are located in between the two primary homoclinic orbits. Furthermore, all the orbits on the unstable branch that lie on the bounded region limited by the curve corresponding to the stable branch 

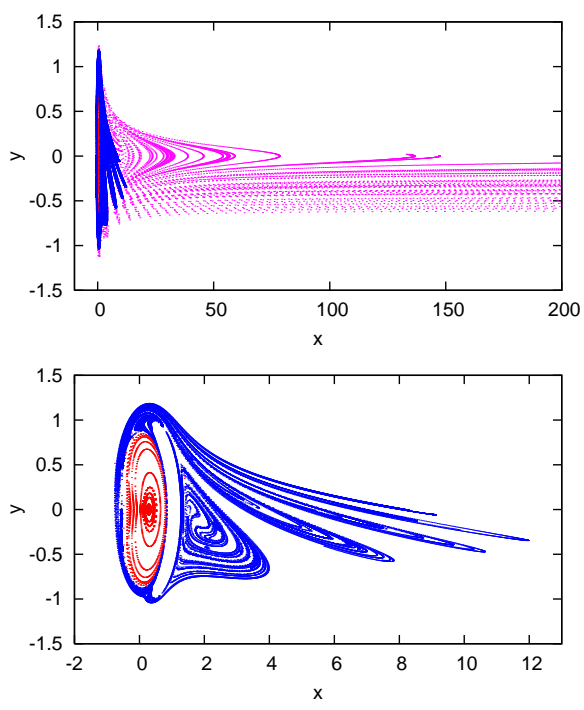

Figure 15: $K=0.1, h=-1.46536975$. (a) Left, PSP showing some of the invariant tori in the confined region of motion, the invariant manifold branch $W_{-}^{u}$ of the LPO of this energy, and some escaping orbits. (b) Right, detail with only the invariant manifold and the invariant tori. In this case, the manifolds are not confined (inside the outermost invariant curve) and they do play a role to explain ionization.

will have a transit passage in the next approach to the LPO. Subsequently, we can know if an orbit has transit passage localizing the point on the plot and its location with respect the stable manifold.

In particular, erratic orbits with large excursions far away from the origin and with close approaches to it can be found. In Figure 17 we show two examples of this behavior. Of course these two orbits provide a simple example of the extraordinarily complicated motion due to the effect of the existence of infinitely many homoclinic orbits. Close to these homoclinic orbits we can find orbits performing a path similar to the homoclinic connections, having passages close to the LPO (with transit or not), but as the invariant manifolds are not confined inside any invariant torus, they can finally ionize, although the time to escape can be very large.

Therefore, we can conclude that for values of $K$ big enough and suitable energy $h$, the invariant manifolds $W^{u}$ and $W^{s}$ become a mechanism to explain a rich variety of ionizing and erratic orbits. 

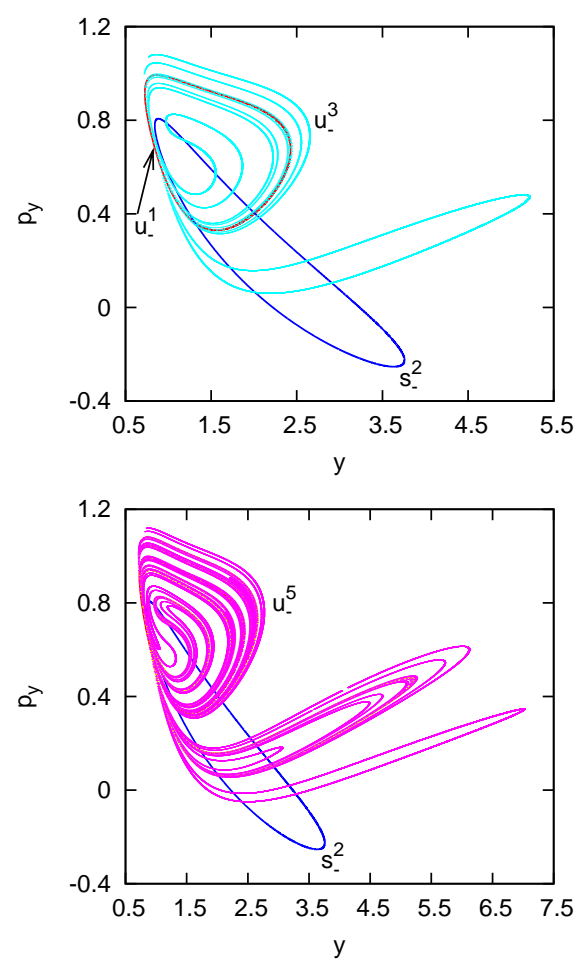

Figure 16: $K=0.1, h=-1.46536975$ and $\Sigma=\{x=1\}$. Intersection curves $u_{-}^{n}$ for (a) $n=1,3$ (left), and also (b) $n=5$ (right) which corresponds to the $n$-th crossings of $W_{-}^{u}$ with $\Sigma$ and curve $s_{-}^{2}$ at the second crossing of $W_{-}^{s}$ with $\Sigma$. The pieces of the curves $u_{-}^{n}$ inside the region bounded by $s_{-}^{2}$ correspond to orbits with transit passages close the LPO.

\section{Quantitative predictions for escape rates}

So far we have discussed some relevant elements of the structure of the phase space, and more particularly those related to ionization/capture.

Our main goal in this Section is to localize and classify the set of initial conditions of ionizing and erratic orbits and to give some experimental estimates of escaping time.

First of all, we recall that, given an orbit of the CP problem, it may remain bounded for all time or become unbounded and escape. In the last case, the electron ionizes (it follows an ionizing orbit). In the process of ionization, we will distinguish two kinds of behavior, that we will call fast and slow ionization.

(i) Fast ionization (FI). An electron has a fast ionization when it follows an orbit that, in rotating coordinates, spirals out toward infinity. The distance to the nucleus increases tending to infinity as time increases, and there is no passage close to the nucleus. In the inertial (non rotating) 

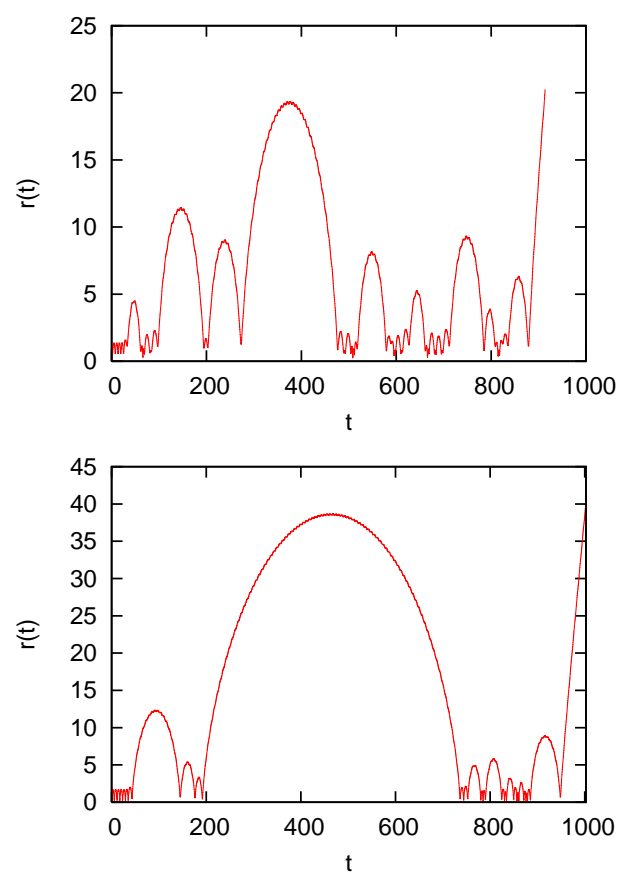

Figure 17: $K=0.1$. Plots of the distance $r(t)$ to the origin of orbits on the unstable branch $W_{-}^{u}$ associated to a LPO with transit passages through the bottleneck around the LPO. (a) Left, $h=-1.46536975$. (b) Right, $h=-1.1$.

coordinates, such an orbit can be approximated by a hyperbolic orbit, so the orbit escapes to infinity.

The dynamics of the FI can be explained from the fact that, when the electron follows an orbit that does not approach the nucleus, its influence can be neglected. Thus, the CP problem can be approximated by a Hamiltonian where the term $1 / r$ has been removed. The new Hamiltonian has associated a linear system of differential equations whose solutions can be expressed as:

$$
\begin{aligned}
& x(t)=C_{1} \cos t+C_{2} \sin t+C_{3}(\cos t+t \sin t)+C_{4} t \cos t+K, \\
& y(t)=-C_{1} \sin t+C_{2} \cos t+C_{3}(-\sin t+t \cos t)-C_{4} t \sin t,
\end{aligned}
$$

for any $C_{i} \in \mathbb{R}, i=1, \ldots, 4$. So, except for the periodic orbits that exist for $h=K^{2} / 2>0$, the electron follows a solution that spirals out and the distance to the nucleus $r(t)$ tends to infinity, both forward and backward in time.

(ii) Slow ionization (SI). An orbit with a slow ionization escapes to infinity very slowly. The orbits exhibit an erratic behavior, that is, in inertial 
coordinates, they consist of a chain of approximated ellipses. Therefore, there are successive approaches to the nucleus (at each turn there is a pericenter passage close to the origin) and the function $r(t)$ does not have a monotone behaviour: on the contrary, it exhibits a sequence of maxima and minima corresponding to each close and farther passage to the nucleus at each revolution around it. For a long enough time, the orbit escapes. We emphasize that the main difference between slowly and fast ionizing orbits is the existence or not of successive close passages to the nucleus.

Actually an ionizing orbit can also exhibit both behaviors, first a SI followed by a FI. However we remark that, if an orbit exhibits a SI for a certain time, then either can continue the same behavior, be a bounded solution, or even escape in a FI after some time. In Figure 18 top we show one example of an orbit exhibiting a SI for a long time, and another one that escapes fast towards infinity after just a couple of revolutions around the nucleus.
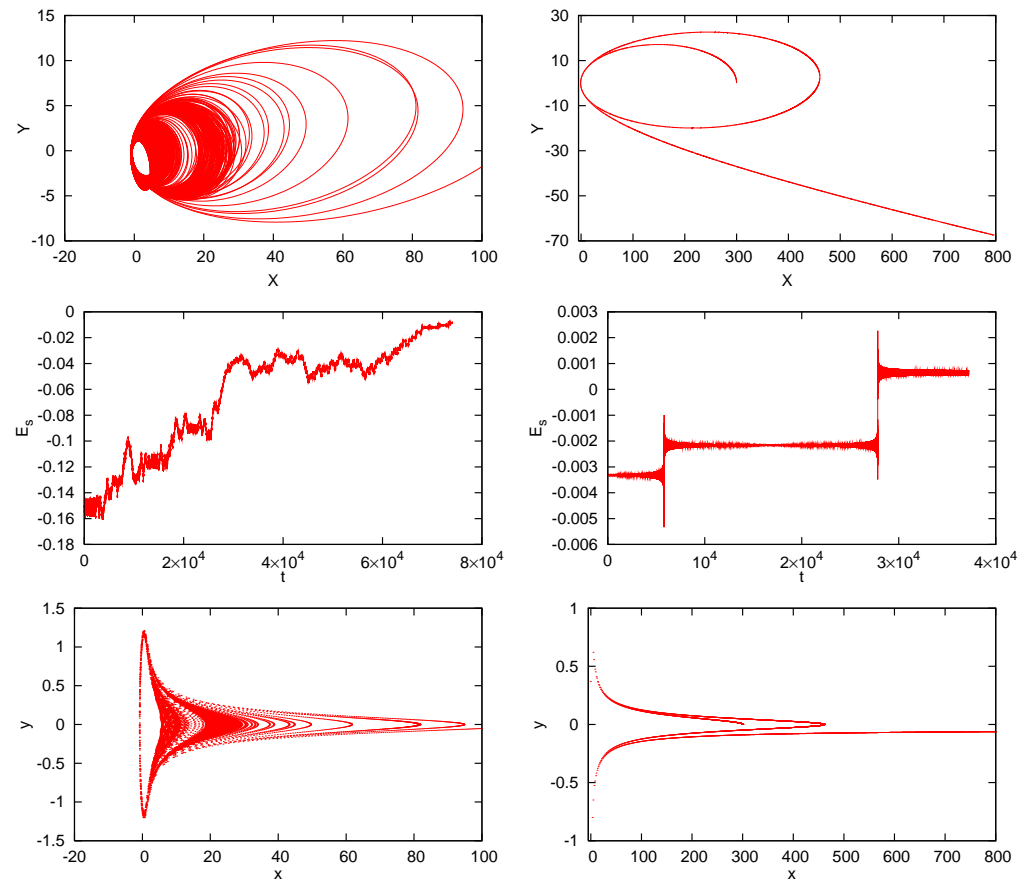

Figure 18: Two orbits of the CP problem for $K=0.0015749$, and $h=-1.4$. Fom top to bottom: the orbit in inertial coordinates $(X, Y)$, the behavior of the function $E_{s}(t)$ and the PSP of each orbit at the section $x^{\prime}=0, y^{\prime}<0$. Left: initial condition $x_{0}=3.8$. Right $x_{0}=300$.

A criterium to decide whether an orbit has a hyperbolic (fast escape) behavior from a particular time $t$ is to check if the osculating sidereal energy $E_{s}(t)$ is positive. At each point, the osculating energy $E_{s}$ is the energy that corresponds 
to the solution of the two-body problem that approximates the orbit, and can be computed from their osculating elements. Since $E_{s}(t)$ shows typically an oscillating character, when varying time, the criterium to decide when an orbit can be approximated by a hyperbola will be that $E_{s}(t)>\delta>0$, for a given small $\delta$. An orbit satisfying this condition will exhibit a FI from this time on.

Given a set of initial conditions, we would like to identify the regions that correspond to bounded and ionizing orbits. From a numerical point of view, the FI behavior is easy to distinguish because the distance to the origin grows continuously (after a given time). However it is difficult, in principle, to distinguish between orbits with a SI and bounded orbits. Furthermore, if an orbit exhibits an erratic behavior (going back and forward to a neighborhood of the origin) for a certain long enough time, for practical purposes we can consider that the orbit does not escape. Therefore we define a new type of orbits that depends on a given (large) distance $D$ and a given (large) time $T$ : the effective bounded erratic orbits, EBE orbits. An orbit exhibits an EBE behavior if while $t \leq T$, it does not reach the distance $D$ from the origin and its osculating energy $E_{s}(t)$ is not positive, so it may be regarded, from a practical point of view, as a bounded orbit, although we do not know if for bigger times the orbit becomes bounded or escapes. Of course a periodic or quasi-periodic orbit orbit will be EBE orbits that will remain bounded for all time.

It is important to emphasize that if an orbit is not an EBE orbit, it does not mean that it ionizes, or on the contrary, an ionizing orbit can be an EBE orbit for a specific time $T$ and distance $D$.

These behaviors are shown in Figure 18, where two different orbits are considered. On the left, we have fixed $D=100$ and $T=10^{5}$. We show an orbit (initial condition at $x_{0}=3.8$ ) with an erratic behavior for a long time (a concatenation of ellipses in the inertial reference frame-top left-, and see also the PSP plot-bottom left-), although it is not an EBE orbit for these values, as it reaches the distance $D$ at time $t=74011.69$. Nevertheless, we do not know if it ionizes: up to time $T$ it exhibits a SI behavior and for the time considered $t \leq 10^{5}$, the osculating energy $E_{s}<0$ (middle plot on the left). On the right plot we have considered a different orbit (initial condition $x_{0}=300$ ) and $D=800$ and $T=5 \times 10^{4}$. In this case, the orbit has a SI behavior for a short period of time, and in fact the osculating energy is negative while $t \lesssim T_{1}=27861$, and then escapes in a FI.

Now in order to get a first insight of the set of initial conditions candidates to give rise to $\mathrm{FI}$ or EBE orbits, let us recall the case $K=0$. In the twobody problem the parabolic orbits (constant sidereal energy $E_{s}=0$ ) are the barrier between the hyperbolic ones $\left(E_{s}>0\right)$-that correspond to FI orbitsand the elliptical ones $\left(E_{s}<0\right)$-bounded orbits-. But for $K \neq 0$, due to the non integrability character of the Hamiltonian, this barrier is not evident at all. However, for small values of the parameter $K$, the two body problem gives a first clue to start the numerical exploration. For $K \neq 0$, those orbits with $E_{s}>0$ (in fact, bigger than a given tolerance) are the natural candidates to FI orbits, whereas those with $E_{s}<0$ are candidates to be EBE orbits, although there will exist orbits with initial $E_{s}<0$ that exhibit an EBE behavior for a 
certain period of time and become FI orbits afterwards, see below.

As we are interested in localizing the initial conditions corresponding to EBE orbits, it is enough to restrict our attention to initial conditions on the positive $y=0$ axis and different velocities. So, given $K \neq 0$, and for each fixed energy value $H=h$, we consider the set of orbits with initial conditions in the rotating frame $\left(x_{0}, y_{0}, x_{0}^{\prime}, y_{0}^{\prime}\right)$ such that $x_{0}>0, y_{0}=0, x_{0}^{\prime}=v \cos \theta, y_{0}^{\prime}=v \sin \theta$, $\theta \in[0,2 \pi)$, where $v$ is determined using the constant energy by

$$
h=\frac{1}{2} v^{2}-\frac{1}{2} x_{0}^{2}-\frac{1}{x_{0}}+K x_{0} .
$$

that comes from Equation (2). Therefore each pair $\left(x_{0}, \theta\right)$ is associated with a unique initial condition and a solution of the CP problem.

At this point, we want to recall that for values of the energy $h$ less that $h_{2}$ (the value of the energy at the equilibrium point $L_{2}$ ), there exist zero velocity curves and a region of forbidden motion (see Figure 1). When the energy is even less than $h_{1}$ (the value corresponding to $L_{1}$ ), the Hill's region (where the motion is allowed in configuration space) has two components, a bounded region around the origin, where the motion is obviously bounded, and an unbounded one. For values of $h \in\left(h_{1}, h_{2}\right)$, the region around the nucleus is not closed (the Hill's region has only one component), so an orbit can escape. But close to the nucleus, the dynamics is strongly governed by its presence and the invariant manifolds associated with the Lyapunov orbits that are born around $L_{1}$. We are interested in the general behavior of the orbits when they are not so close to the nucleus, so we focuss our attention on initial conditions located at the "right-hand side" of the zero velocity curve, that is, with $x_{0}>x_{c}>0$, being $x_{c}$ the farthest intersection of the zero velocity curve with $y=0$ (in configuration space).

So, for each fixed energy $h$, we consider the region $\left\{\left(x_{0}, \theta\right) \in\left[x_{m}, \infty\right) \times\right.$ $[0,2 \pi)\}$, where $\theta=0$ and $\theta=2 \pi$ are identified, and $x_{m}$ is a suitable positive value not close to the origin (typically $x_{m} \geq x_{c}$ when zero velocity curves exist, but bigger values of $x_{m}$ can be considered). The solution of the CP problem can be approximated by a solution of the two-body problem with initial osculating sidereal energy $E_{s}^{0}$

$$
E_{s}^{0}=\frac{1}{2}\left(X^{\prime 2}+Y^{\prime 2}\right)-\frac{1}{\sqrt{X^{2}+Y^{2}}}=\frac{v^{2}}{2}+\frac{x_{0}^{2}}{2}+x_{0} v \sin \theta-\frac{1}{x_{0}},
$$

where $X=x_{0}, Y=0, X^{\prime}=v \cos \theta, Y^{\prime}=x_{0}+v \sin \theta$ and $v$ comes from Equation (3). In particular we have that $E_{s}^{0}=0$ if and only if

$$
\sin \theta=\frac{-h-x_{0}^{2}+K x_{0}}{v x_{0}} .
$$

This expression gives a curve inside the region of the $\left(x_{0}, \theta\right)$ plane considered (see Figure 19). This curve delimits what we call the erratic region $R$, defined as the set of points $\left(x_{0}, \theta\right)$ for which $E_{s}^{0}<0$. The initial conditions inside this 
region will be the natural candidates to correspond to EBE orbits. Clearly, as we are using the two body problem as an approximation, this is valid only for small values of the parameter $K$.

We want to stress some properties of the shape of the erratic region $R$. First, recall that we are looking into the right hand side of the zero velocity curve (when it exists), that is, $x_{0}>x_{m}$. Second, let $f(x)$ be the function on the right hand side of Equation (4). The region $R$ corresponds to those points such that $\sin \theta<f(x)$. It can be shown that if $h<-1 /(2 K), f(x)<-1$, and the region $R$ is empty. When $h>-1 /(2 K)$, we have that

(a) $f(x)^{2}=1$ has exactly two solutions,

$$
\tau_{1,2}=\frac{1+K h \pm \sqrt{1+2 K h}}{K^{2}},
$$

and $|f(x)|<1$ for $x \in\left(\tau_{1}, \tau_{2}\right)$. So the region $R$ is bounded.

(b) As $x_{0}$ grows, the shape of the region is like a "spear" symmetric with respect $\theta=3 \pi / 2$. This is due to the fact that $f(x)$ tends to -1 very quickly.

In Figure 19 the shape of the region $R$ is shown, for different values of the energy. For example, for the value $K=0.0015749$, the minimum value of the energy for which $R$ is not empty is -317.480475 . We are not interested at all in such big negative values of the energy (in this case, also the forbidden region is huge). We will explore moderate values of the energy, say $h \geq-2$. For these values, the last point for which the erratic region exists is of order $2 / K^{2} \simeq 4.03175 \times 10^{5}$, so although $R$ is bounded, we can still find orbits with initial conditions far away from the nucleus and with negative osculating energy.

Of course the boundary of $R$ is not really a barrier that separates erratic orbits from FI behavior, but it (somewhat) shows where the transition between the two behaviors occurs.

Then, fixed a value of the energy $h$, a distance from the origin $D$ and a time $T$, we explore the region of the $\left(x_{0}, \theta\right)$ plane with $x_{0}<D$, and proceed in two steps as follows. For each fixed $x_{0}$ :

1. the curve (4) gives an interval (or several intervals) of values of $\theta, I_{x_{0}}=$ $\left[a_{x_{0}}, b_{x_{0}}\right]$ for which $E_{s}^{0}<0$. Since $E_{s}^{0}=0$ is not exactly a barrier, we will explore values of $\theta \in I_{x_{0}, \epsilon}=\left[a_{x_{0}}-\epsilon, b_{x_{0}}+\epsilon\right]$ with a constant $\epsilon>0$. If there exists more than one interval, we repeat the procedure in all of them.

2. We consider a set of values in $I_{x_{0}, \epsilon}$, an for each one we integrate the corresponding initial conditions and we apply the numerical criterion to identify EBE orbits.

We performed several explorations for $K=0.0015849, h=-1.4$ and $x \in$ $[3,10]$. We fixed $D=100$ and considered two different final times: $T=5 \times 10^{4}$ and $T=10^{5}$. The total number of values of $\theta \in[0,2 \pi]$ is fixed, although we 

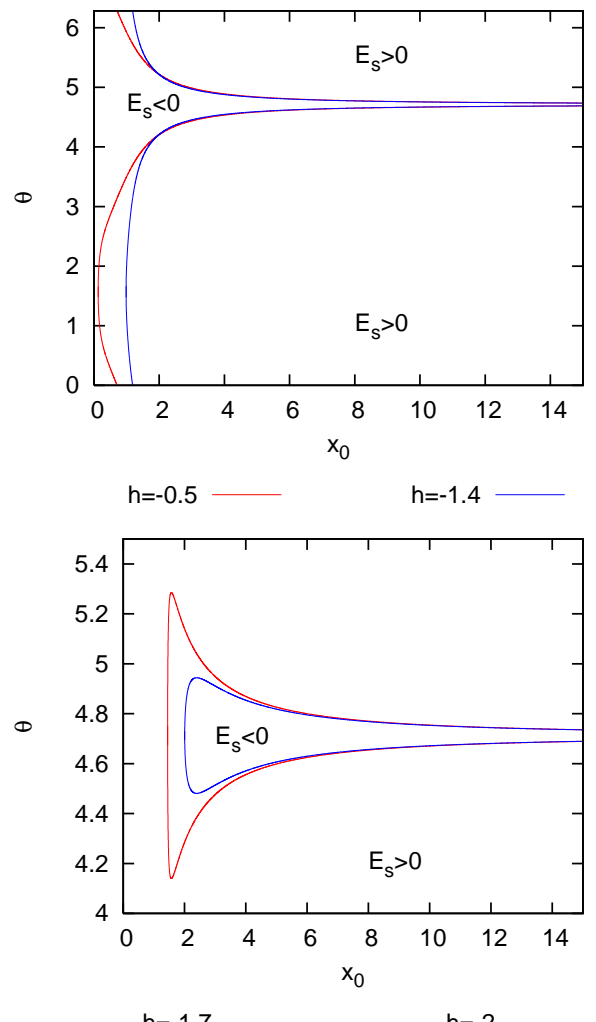

$\mathrm{h}=-1.7 \longrightarrow \mathrm{h}=-2$

Figure 19: For $K=0.0015749$, curves of initial conditions $\left(x_{0}, \theta\right)$ with initial osculating energy $E_{s}^{0}=0$ for the values $h=-2,-1.7,-1.4,-0.5$.

focus our attention in the values corresponding to the interval $I_{x_{0}, \epsilon}$ for each initial $x_{0}$. We classify the orbits as EBE or FI depending if the two conditions (not escaping and negative sidereal energy) are both satisfied, or none of them respectively. If only one of the conditions fail, we are in a kind of intermediate state: it can happen that an orbit reaches the distance $D$ before the final time, but its sidereal energy $E_{s}$ is negative, or the contrary, the orbit does not escape for $t<T$, but it reaches the final time with positive sidereal energy. In the first case, we cannot be sure about the future behavior of the orbit, it can be an EBE orbit for a bigger value of $D$ or $T$, or escape, whereas if an orbit exhibits positive $E_{s}$ we can predict that it will escape. In fact, we have not found any orbit of the last case. Therefore, we will show the results corresponding to EBE orbits, FI orbits and orbits that reach the distance $D$ for $t<T$ with $E_{s}<0$.

In the explorations done, and as we expected, the EBE orbits are located inside the erratic region. In Figure 20 we show the other two types of orbits, and the curve corresponding to $E_{s}^{0}=0$. The FI behavior (in green) is located 
outside the erratic region, and the orbits that cannot be classified as EBE or FI orbits for these specific values of $T$ and $D$ are located around that curve, and inside the erratic region. As it was predicted from the two body problem, we can see how the set of initial conditions corresponding to EBE orbits shrinks. We can also observe how when we increase the final time, there is a significant amount of orbits that were classified previously as EBE orbits for the smaller final time and for the new final time they reach the distance $D$. Nevertheless, all of these orbits still have negative sidereal energy, so we can expect them to act as EBE orbits still for some time.
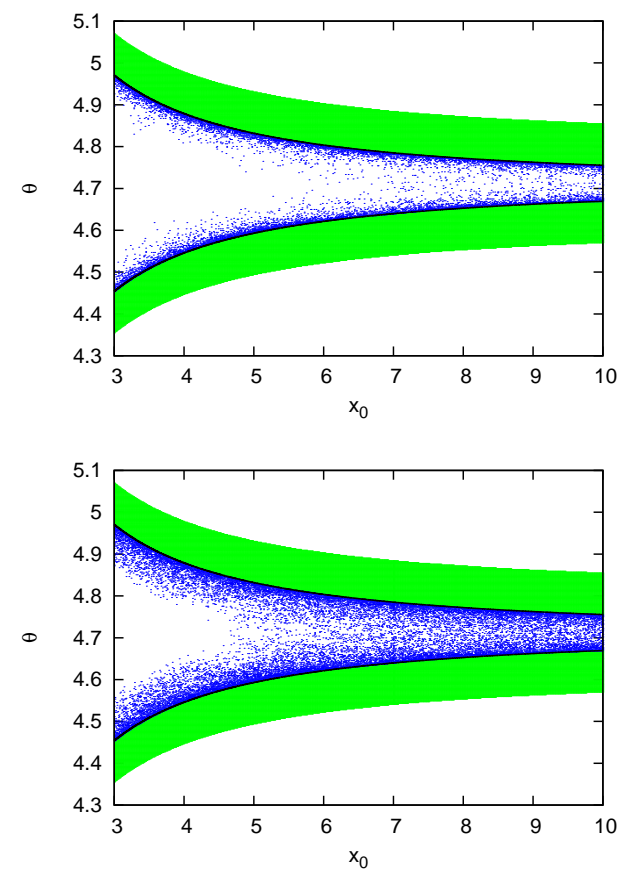

Figure 20: For $K=0.0015749, h=-1.4, D=100$, and $T=5 \times 10^{4}$ (left) and $T=10^{5}$ (right), the plots show the points that correspond to initial conditions of orbits that cannot be considered EBE for these values of $T$ and $D$, but they still have negative $E_{s}$ (in blue), and FI orbits (in green). The black line corresponds to the curve $E_{s}^{0}=0$.

In fact, we can measure the ratio of EBE orbits for each initial $x_{0}$. We count the number of EBE orbits and the number of orbits that reach the distance $D$ but have negative $E_{s}$. In Figure 21 such ratios (maximum 1) are plotted. We can see that for $x_{0}=3$ these orbits are below $8 \%$ of the total, whereas for $x_{0}=10$ they are less than $1 \%$.

The same kind of explorations have been done for other values of $h$ obtaining similar results. We show in Figure 22 the plot of the ratios obtained when taking 

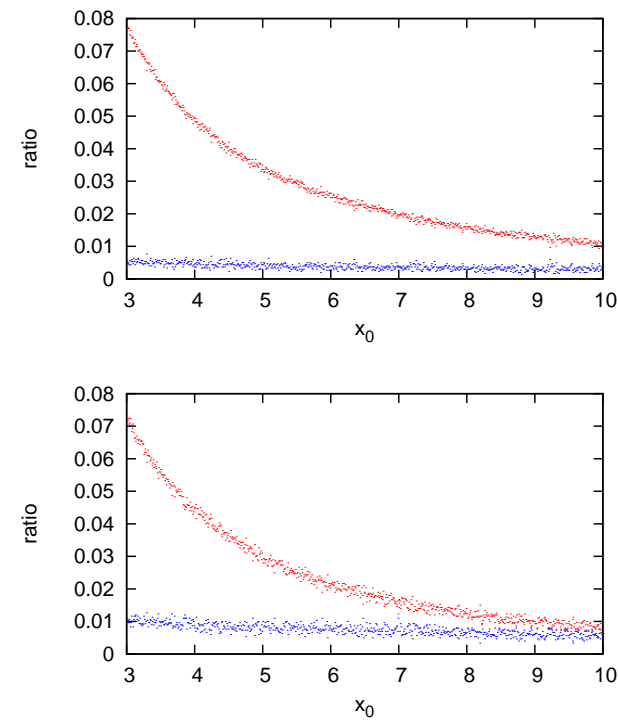

Figure 21: For $K=0.0015749, h=-1.4, D=100$ and $T=5 \times 10^{4}$ (left) and $T=10^{5}$ (right), the plots show the ratio of initial conditions of EBE orbits (in red) and the ratio of orbits that can not be considered EBE for these values of $T$ and $D$, but they still have negative $E_{s}$, when reaching the distance $D$ (in blue).

$D=100$ and $T=5 \times 10^{4}$ for $h=-1.7$ and $h=-0.557$.

In fact the existence of the stable retrograde periodic orbit of family $r_{i}$ (see Figure 10 for $h=-1.7$ and 13 for $h=-0.557$ ) is the responsible for the existence of the invariant curves and the erratic region for the region of EBE orbits. In particular, the bigger the $x_{0}$ (the smaller the interval of $\theta$ such that the associated initial condition satisfies $E_{s}^{0}<0$ ), the larger the number of orbits that reach the distance $D$ and this agrees with the behaviour of the chaotic orbits shown in the PSP of Figures 10 and 13.

From the numerical results we can conclude that for a small $K \neq 0$ :

- Given a neighborhood $U$ of the origin, of moderate radius $r$, if an orbit does not enter $U$, it typically describes a fast ionizing orbit. For each $h$, the set of $\left(x_{0}, \theta\right)$ exhibiting FI has relative big measure (with respect to $\theta \in[0,2 \pi))$.

- For any given energy, there exists a small (bottleneck shaped) erratic region in $\left(x_{0}, \theta\right)$, thinner as $x_{0}$ grows, containing initial conditions of EBE orbits. Moreover, the ratio of EBE orbits (which is typically bigger than the ratio of orbits that reach $D$ for $t<T$ with $E_{s}<0$ ), becomes smaller when $x_{0}$ grows. See Figures 21 and 22 . 

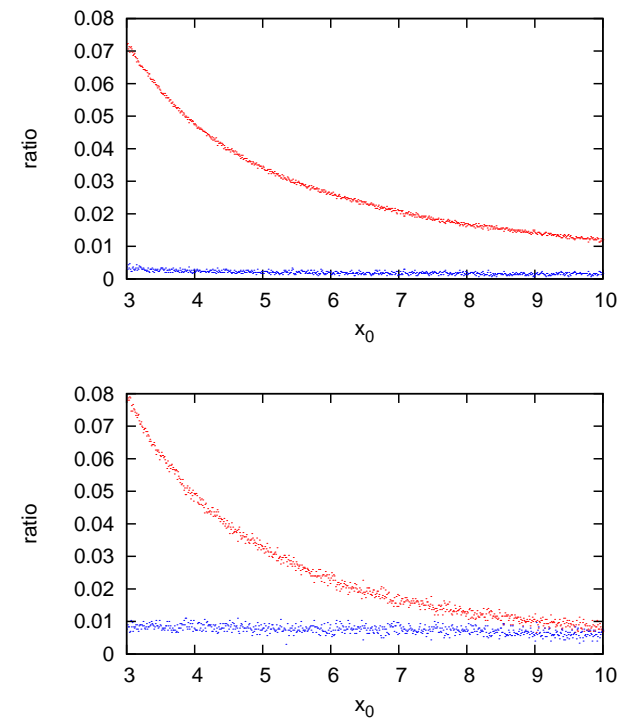

Figure 22: For $K=0.0015749, D=100, T=5 \times 10^{4}$ (left) and $h=-1.7$ (left), $h=-0.557$ (right), the plots show the ratio of initial conditions of EBE orbits (in red) and the ratio of orbits that can not be considered EBE for these values of $T$ and $D$, but they still have negative $E_{s}$, when reaching the distance $D$ (in blue).

- Since the erratic region $R$ is bounded, there is a maximum value $x_{0, M} \approx$ $\frac{2}{K^{2}}$ such that, for $x_{0}>x_{0, M}$, any initial condition $\left(x_{0}, 0, x^{\prime}, y^{\prime}\right)$ gives rise to a FI orbit. However $x_{0, M}$ is very big, so we can also find large values of $x_{0}$ and values of $\theta$ close to $3 \pi / 2$ (from (4)) which are the natural candidates for initial conditions of EBE orbits. See for example Figure 18 right with $x=300$ and $\theta=4.712389$ and Figure 20.

- The stable orbit of family $r_{i}$, its surrounding tori as well as the fact that our problem is close to an integrable one play an important role in the dynamics. So we have the simultaneous presence of regular (periodic and quasiperiodic) orbits as well as regions of stochasticity.

\section{Conclusions}

We have considered the hydrogen atom in circular polarized microwave fields, paying special attention to in the ionization process. The problem has been attacked with an strategy combining two points of view: as a perturbed Kepler problem and as a 2 degree of freedom Hamiltonian problem where the standard tools of dynamical systems can be applied. 
For small $K>0$, the ionizing/escaping orbits either come from perturbed non-bounded orbits of the $K=0$ case, whose corresponding $(x, y)$ projection spirals fast outwards to infinity, or they are erratic orbits, that is, during its journey to ionization, the electron undergoes erratic motions (making several passages close to the origin), resulting in 'slowly' ionizing trajectories (finally escaping to infinity).

The dominant channel for escape for moderate electric field values is due to 'diffusive' motion in KAM tori region in the outer part of the phase space. Phase space transport from the inner region due to stable/unstable manifold mechanism only plays a role for fairly large field strengths. Actually, the mentioned manifolds for very small $K$ are situated inside the domain bounded by the outermost invariant KAM-curve and thus do not play any role in the ionization.

Through the analysis of the homoclinic connections, we have explained the path followed by the electron to leave and possibly return (many times) to the nucleus giving rise to bounded or ionizing orbits.

However not all initial conditions lead to ionization, so we have analysed and classified several areas of phase space and some quantitative (experimental) estimates for the escape rates have also been discussed.

\section{Acknowledgements}

Useful discussions with C. Simó are gratefully acknowledged. The first author was partially supported by grants MTM2006-05849/Consolider and MTM201016425. The second author was partially supported by grants MCyT/FEDER MTM2009-0697 and 2009SGR859. The third author was partially supported by grants iMath CSD2006-32 and MTM2009-14621. The fourth author was partially supported by NSF(USA). The fifth author was partially supported by grants MTM2005-02139 and MTM2006-05849/Consolider.

\section{Appendix. Equations and basic features of the CP problem}

In this Section, we give some details with respect to the equations and some features of the CP problem. We consider the Hamiltonian given by (1). We restrict our attention to solutions contained in the plane $z=0$ (planar problem), so we omit the variables $z$ and $p_{z}$. Moving to a frame rotating with the CP field (with angular velocity $w$ ), the Hamiltonian becomes autonomous and its expression is

$$
H_{1}=\frac{1}{2}\left(p_{x}^{2}+p_{y}^{2}\right)-\omega\left(x p_{y}-y p_{x}\right)-\frac{1}{r}+F x,
$$

where now $(x, y)$ and $\left(p_{x}, p_{y}\right)$ correspond to positions and momenta in the rotating frame. This Hamiltonian can be simplified by re-scaling time and distances. 
Defining a new time $s=w t$ and introducing the symplectic change of coordinates with multiplier $a^{2} w$

$$
(x, y)=a(\widetilde{x}, \widetilde{y}), \quad\left(p_{x}, p_{y}\right)=a w\left(\widetilde{p}_{x}, \widetilde{p}_{y}\right),
$$

the expression of the Hamiltonian in the new variables, after dropping the tilde, is

$$
H_{2}=\frac{1}{2}\left(p_{x}^{2}+p_{y}^{2}\right)-x p_{y}+y p_{x}-\frac{1}{r}+K x,
$$

where $K=F / w^{4 / 3}>0$ and $a$ has been chosen such that $a^{3} w^{2}=1$. The relation between both Hamiltonians is $H_{2}=H_{1} / w^{2 / 3}$, and $H_{2}$ is in fact the Hamiltonian $H$ (2) of the CP problem. Clearly, the change performed does not have sense if $w=0$. We remark that for $K=0$ this Hamiltonian corresponds to the two body problem.

The equations of the motion of the CP problem (2) are

$$
\begin{aligned}
x^{\prime} & =p_{x}+y, \\
y^{\prime} & =p_{y}-x \\
p_{x}^{\prime} & =p_{y}-\frac{x}{r^{3}}-K, \\
p_{y}^{\prime} & =-p_{x}-\frac{y}{r^{3}}
\end{aligned}
$$

which satisfy the symmetry

$$
\left(t, x, y, p_{x}, p_{y}\right) \longrightarrow\left(-t, x,-y,-p_{x}, p_{y}\right)
$$

This implies that, for each solution of the equations of motion, there also exists another one which is symmetric with respect to $y=0$ in configuration space. In particular, periodic symmetric solutions with respect to $y=0$ intersect this axis perpendicularly twice. We use this property to find families of periodic symmetric solutions.

Using the equations of motion (5) the equilibrium points can be found as the solutions of the system

$$
\begin{array}{r}
x-\frac{x}{r^{3}}-K=0, \\
y-\frac{y}{r^{3}}=0 .
\end{array}
$$

The second equation is satisfied only when $y=0$ or $r=1$. If we substitute $r=1$ in the first equation we have $K=0$, which corresponds to the two body problem. Restricting our attention only to $K>0$, then $y=0$ and the $x$ coordinate of an equilibrium point has to satisfy

$$
f(x)=x^{3}-K x^{2}-\operatorname{sign}(x)=0,
$$

where $\operatorname{sign}(x)$ stands for the sign of $x$.

Lemma 1 The problem given by the Hamiltonian (2) has only two equilibrium points located at $\left(x_{i}, 0\right), i=1,2$ with the following properties:

1. $\max (-1,-1 / \sqrt{K}) \leq x_{1}<0$ and $x_{2}>\max (1,2 K / 3)$; 
2. $\lim _{K \rightarrow 0} x_{1}=-1$ and $\lim _{K \rightarrow 0} x_{2}=1$;

3. both $x_{i}, i=1,2$, are increasing functions of $K$.

Proof. On one side, the function $f(x)$ defined in (7), increases in $(-\infty, 0)$, $f(-1)=-K<0 f(-1 / \sqrt{K})=-K^{-3 / 2}<0$ and $\lim _{x \rightarrow 0-} f(x)=1$. On the other side, the function $f(x)$ decreases over $(0,2 K / 3)$, increases in $(2 K / 3,+\infty)$, $f(1)=-K<0$ and $f(2 K / 3)<0, \lim _{x \rightarrow 0+} f(x)=-1, \lim _{x \rightarrow \infty} f(x)=\infty$. Thus, there only exists two solutions of $f(x)=0, x_{1}<0<x_{2}$ satisfying the properties of the first statement.

Second statement comes directly from the continuity of $x_{i}, i=1,2$ with respect $K$. Finally, for each $i=1,2$, deriving implicitly the equation $f\left(x_{i}\right)=0$ with respect to $K$ we have that $x_{i}$ are increasing functions of $K$.

We call $L_{i}$ the equilibrium point located at $\left(x_{i}, 0\right), i=1,2$ and we denote by $h_{i}=H\left(L_{i}\right), i=1,2$ the energy at each equilibrium point. In Table 1 the position and energy of the equilibrium points for two different values of the parameter $K$ are shown (the ones mainly used through the paper).

\begin{tabular}{|l|c|c|}
\hline & $L_{1}$ & $L_{2}$ \\
\hline \multirow{2}{*}{$K=0.0015749$} & $x_{1}=-0.99947531$ & $x_{2}=1.00052524$ \\
\cline { 2 - 3 } & $h_{1}=-1.50157449$ & $h_{2}=-1.49842469$ \\
\hline \multirow{2}{*}{$K=0.1$} & $x_{1}=-0.96775311$ & $x_{2}=1.03446911$ \\
\cline { 2 - 3 } & $h_{1}=-1.59836975$ & $h_{2}=-1.39829568$ \\
\hline
\end{tabular}

Table 1: Equilibrium points: position ( $x$ coordinate) and energy for two different values of $K$

The linear stability of each equilibrium point is stated in the following Lemma.

Lemma 2 Let $L_{1}, L_{2}$ be the equilibrium points of the problem given by the Hamiltonian (2). Then, for $K>0$

1. $L_{1}$ is of type center $\times$ saddle for all values of $K$.

2. $L_{2}$ is of type center $\times$ center for $K \leq \frac{3^{-4 / 3}}{2} \simeq 0.11556021$ and is a complex saddle for $K>\frac{3^{-4 / 3}}{2}$.

Proof. The eigenvalues associated to each equilibrium point can be easily calculated in terms of the first coordinate of each point $x_{i}, i=1,2$. In the case of $L_{1}$, the eigenvalues are $\pm \lambda_{i}, i=1,2$, where

$$
\lambda_{1}^{2}=\frac{2 x_{1}^{3}+1+\sqrt{8 x_{1}^{3}+9}}{2\left|x_{1}\right|^{3}}, \quad \lambda_{2}^{2}=\frac{2 x_{1}^{3}+1-\sqrt{8 x_{1}^{3}+9}}{2\left|x_{1}\right|^{3}} .
$$

It can be proved that $\lambda_{1}^{2}>0$ and $\lambda_{2}^{2}<0$ for all values of $K$, so that $L_{1}$ is of type center $\times$ saddle. 
In the case of $L_{2}$, the eigenvalues are $\pm \mu_{i}, i=1,2$, where

$$
\mu_{1}^{2}=\frac{1-2 x_{2}^{3}+\sqrt{9-8 x_{2}^{3}}}{2 x_{2}^{3}}, \quad \mu_{2}^{2}=\frac{1-2 x_{2}^{3}-\sqrt{9-8 x_{2}^{3}}}{2 x_{2}^{3}} .
$$

It can be seen that for $K>\frac{3^{-4 / 3}}{2}$, the discriminant $9-8 x_{2}^{3}<0$, and for $K \leq \frac{3^{-4 / 3}}{2}$, both $\mu_{i}^{2}<0, i=1,2$.

Finally, we examine the topology of the region in configuration space where the motion is possible, called Hill's region following the naming in celestial mechanics. In particular we are interested in how the zero-velocity curves (that is, the boundary of the Hill's regions) evolve as a function of $K$ and the energy. Notice that, from the first statement of Lemma 1, the equilibrium points are such that $\lim _{K \rightarrow+\infty} x_{1}=0$ and $\lim _{K \rightarrow+\infty} x_{2}=+\infty$.

The zero velocity curve (zvc) is obtained from Hamiltonian (2). For a fixed value of the energy $H=h$, the modulus of the velocity $v=\sqrt{x^{\prime 2}+y^{\prime 2}}$ is given by

$$
v^{2}=2 h+r^{2}+\frac{2}{r}-2 K x .
$$

Thus, the zero velocity curve for a fixed energy level $h$ is defined implicitly by

$$
r^{2}+\frac{2}{r}-2 K x=-2 h
$$

or equivalently, $(x-K)^{2}+y^{2}+\frac{2}{r}=K^{2}-2 h$. We notice the following:

- For $h<0$ with large $|h|$, the region of motion has two components: a bounded one, near the origin and inside a closed curve close to the circle $r=-1 / h$ (inner component), and an unbounded one, outside a closed curve similar to the circle of center $(K, 0)$ and radius $\sqrt{K^{2}-2 h}$ (outer component). See Figure 1, (a).

- For $h=h_{1}$ the two zero velocity curves intersect at $L_{1}$ and for $h>h_{1}$, the region of motion has only one component, and the inner and outer regions are connected via a bottleneck region around the equilibrium point $L_{1}$. See Figure 1, (b) and (c).

- The maximum value of $h$ for which a zero velocity curve exists is $h_{2}$. After this value all the plane is available for the motion.

\section{References}

[1] S.A. Astakhov, E.A. Lee and D. Farrelly. Formation of Kuiper-belt binaries through chaos-assisted capture. MNRAS, 360:401-415, 2005.

[2] E. Barrabés, J. M. Mondelo, and M. Ollé. Dynamical aspects of multiround homoclinic orbits in the RTBP. Cel. Mech. and Dynam. Astron., 105(1):197-210, 2009. 
[3] E. Barrabés, J. M. Mondelo, and M. Ollé. Numerical continuation of families of homoclinic connections of periodic orbits in the RTBP. Nonlinearity, 22(12):2901-2918, 2009.

[4] M. R. W. Bellermann, P. M. Koch and D. Richards. Resonant, ellipticalpolarization control of microwave ionization of hydrogen atoms. Phys. Rev. Lett., 738(20):3840-3843, 1997.

[5] I. Bialynicki-Birula, M. Kalinsi, and J. Eberly. Lagrange Equilibrium Points in Celestial Mechanics and Nonspreading Wave Packets for Strongly Driven Rydberg Electrons Phys. Rev. Lett., 73:1777-1789, 1994.

[6] A. F. Brunello, T. Uzer, and D. Farrelly. Hydrogen atom in circularly polarized microwaves: Chaotic ionization via core scattering. Phys. Rev. A, 55(5):3730-3745, 1997.

[7] A. Buchleitner, D. Delande and J. Gay. Microwave ionization of threedimensional hydrogen atoms in a realistic numerical experiment. J. Opt. Soc. Am. B, 12(4)505-519, 1995.

[8] C. Conley. Low energy transit orbits in the restricted three-body problem. SIAM Journal on Applied Mathematics, 16(4):732-746, 1968.

[9] R. L. Devaney. Blue sky catastrophes in reversible and Hamiltonian systems. Indiana Univ. Math. J., 26(2):247-263, 1977.

[10] M. C. Gutzwiller. Moon-Earth-Sun: The oldest three-body problem. Rev. Mod. Phys., 70(2):589-639, 1998.

[11] C. Jaffé, D. Farrelly, and T. Uzer. Transition state theory without timereversal symmetry: Chaotic ionization of the hydrogen atom. Phys. Rev. Lett., 84(4):610-613, 2000.

[12] A. Jorba and M. Zou. A software package for the numerical integration of ODEs by means of high-order Taylor methods. Experimental Mathematics, 14:99-117, 2005.

[13] P. M. Koch, K.A.H. van Leeuwen. The importance of resonances in microwave ionization of excited hydrogen atoms. Phys. Reports, 255:289-403, 1995.

[14] L. M. Lerman. Hamiltonian systems with loops of a separatrix of a saddlecenter [translation of methods of the qualitative theory of differential equations (Russian), 89-103, Gor'kov. Gos. Univ., Gorki, 1987; MR0987443 (90g:58036)]. Selecta Math. Soviet., 10(3):297-306, 1991. Selected translations.

[15] J. Llibre. On the restricted three-body problem when the mass parameter is small. Cel. Mech. and Dynam. Astron., 28:83-105, 1982. 
[16] H. Maeda and T. F. Gallagher. Nondispersing wave packets. Phys. Rev. Lett., 92(13):133004, 2004.

[17] F. Mauger, C. Chandre and T. Uzer. Recollisions and correlated double ionization with circularly polarized light. Phys. Rev. Lett., 105, 083002, 2010 .

[18] K. R. Meyer and G. R. Hall. Introduction to Hamiltonian dynamical systems and the $N$-body problem, vol. 90 Applied Mathematical Sciences. SpringerVerlag, New York, 1992.

[19] T. Petrosky. Chaos and cometary clouds in the solar system. Physics Letters, 117:328-332, 1986.

[20] C. Simó and T. J. Stuchi. Central stable/unstable manifolds and the destruction of KAM tori in the planar hill problem. Physica D, 140(1-2):1-32, 2000.

[21] T. Uzer, C. Jaffé, J. Palacián, P. Yanguas, and S. Wiggins. The geometry of reaction dynamics. Nonlinearity, 15(4):957-992, 2002.

[22] H. Waalkens, A. Burbanks, and S. Wiggins. Phase space conduits for reaction in multidimensional systems: HCN isomerization in three dimensions. J. Chem. Phys., 121(13):6207-6225, 2004.

[23] J. Zakrzewski, D. Delande and J. Gay. Ionization of highly excited hydrogen atoms by a circularly polarized microwave field. Phys. Rev. A, 47(4):24682471, 1993. 